\title{
Constant Curvature Algebras and Higher Spin Action Generating Functions
}

\author{
K. Hallowell ANd A. WALdRon \\ Department of Mathematics, University of California, Davis CA 95616, USA \\ hallowell, wally@math.ucdavis.edu
}

\begin{abstract}
The algebra of differential geometry operations on symmetric tensors over constant curvature manifolds forms a novel deformation of the $\operatorname{sl}(2, \mathbb{R}) \ltimes \mathbb{R}^{2}$ Lie algebra. We present a simple calculus for calculations in its universal enveloping algebra. As an application, we derive generating functions for the actions and gauge invariances of massive, partially massless and massless (for both bose and fermi statistics) higher spins on constant curvature backgrounds. These are formulated in terms of a minimal set of covariant, unconstrained, fields rather than towers of auxiliary fields. Partially massless gauge transformations are shown to arise as degeneracies of the flat, massless gauge transformation in one dimension higher. Moreover, our results and calculus offer a considerable simplification over existing techniques for handling higher spins. In particular, we show how theories of arbitrary spin in dimension $d$ can be rewritten in terms of a single scalar field in dimension $2 d$ where the $d$ additional dimensions correspond to coordinate differentials. We also develop an analogous framework for spinor-tensor fields in terms of the corresponding superalgebra.
\end{abstract}




\section{Contents}

1 Introduction 3

2 Constant Curvature Algebra $\quad 6$

2.1 Algebra of Operations . . . . . . . . . . . . . . . . 9

2.2 Analysis of the Operator Algebra . . . . . . . . . . . . 10

2.2.1 Normal Ordering and Hadamard Products . . . . . . . 12

2.2.2 Casimir Basis . . . . . . . . . . . . . . . . . 13

3 Generating Function for Massless Higher Spin Actions 15

4 Radial Dimensional Reduction $\quad 17$

5 Generating Functions for Massive Actions 18

5.1 Radially Reduced Gauge Variations . . . . . . . . . . . . . . . 20

5.2 Analysis of Gauge Transformations . . . . . . . . . . . . . . 21

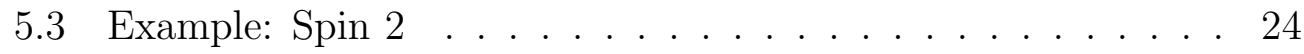

5.4 Radially Reduced Action . . . . . . . . . . . . . . 26

6 Partially Massless Theories 28

$\begin{array}{lll}7 & \text { Fermions } & 31\end{array}$

7.1 Generating Function for Massless Fermion Actions . . . . . . . 35

8 Scalar Fields on the Total Space 36

$\begin{array}{llr}9 & \text { Conclusions } & 37\end{array}$

$\begin{array}{ll}\text { A The Constant Curvature Algebra } & 38\end{array}$

A.1 Non-Commutative Harmonic Oscillators . . . . . . . . . . . . 38

A.2 Higher Dimensional Embedding . . . . . . . . . . . . . . . 39

A.3 Two Component Notation . . . . . . . . . . . . . . . . . 40

B On-shell Partially Massless Fields $\quad 41$ 


\section{Introduction}

The central objects of our study are sections of the symmetric tensor bundle $\odot T^{*} M$ for constant curvature manifolds $M$. In physics parlance, these are totally symmetric tensor fields whose most common application is to theories of higher spin particles, and our physical motivation is to provide a formulation of these theories in terms of a simple calculus of operators acting on symmetric tensors ${ }^{1}$. The simplicity of the algebra we find suggests that totally symmetric tensors, just like their totally antisymmetic differential form counterparts, may be useful tools for mathematical studies of constant curvature manifolds.

Our main results are rather simple, so we summarize them here in the Introduction, leaving detailed derivations, explanations and comments to the ensuing Sections of this Article:

A first and key step is to introduce the index operator $\mathbf{N}$ whose eigenvectors are sections $\Phi \in \odot T^{*} M$ with a definite number of indices, i.e.

$$
\mathbf{N} \Phi=s \Phi \quad \Longrightarrow \quad \Phi=\varphi_{\mu_{1} \ldots \mu_{s}} d x^{\mu_{1}} \ldots d x^{\mu_{s}}
$$

where $d x^{\mu_{1}} \ldots d x^{\mu_{s}} \equiv \frac{1}{s !} \sum_{\sigma} d x^{\mu_{\sigma(1)}} \otimes \cdots \otimes d x^{\mu_{\sigma(s)}}$. This is in keeping with our physics goal of writing generating functions for theories of arbitrary spin $s$.

Next we introduce operators $\mathbf{g}, \mathbf{t r}: \odot T^{*} M \rightarrow \odot T^{*} M$ which act on eigenvectors of $\mathbf{N}$ by multiplication by the metric and symmetrizing, and contraction of a pair of indices, respectively. The triplet $\{\mathbf{g}, \mathbf{N}, \mathbf{t r}\}$ generate the Lie algebra $\operatorname{sl}(2, \mathbb{R})$. The doublet formed from the gradient and divergence operators $\{\mathbf{g r a d}, \mathbf{d i v}\}$ then transform as the fundamental representation. Were these operators to commute, we would be faced with the Lie algebra $\operatorname{sl}(2, \mathbb{R}) \ltimes \mathbb{R}^{2}$, however, instead we have a deformation thereof because on a constant curvature manifold,

$$
[\operatorname{div}, \operatorname{grad}]=\square-2 \mathrm{c} .
$$

This relation completes our "constant curvature algebra". The operator is central and related to the usual Laplacian $\Delta$ by

$$
\square=\Delta+\mathbf{c}=[\operatorname{div}, \operatorname{grad}]+2 \mathbf{c},
$$

\footnotetext{
${ }^{1}$ The tensorial side of higher spin computations is necessarily complicated. For a discussion of some of the difficulties involved, see [1].
} 
and

$$
\mathbf{c}=\mathbf{g} \mathbf{t r}-\mathbf{N}(\mathbf{N}+n-1)
$$

is the $\operatorname{sl}(2, \mathbb{R})$ Casimir while $n \equiv \operatorname{dim}(M)-1$. Note that $\square$ is precisely the Lichnerowicz wave operator [2]. Also the appearance of the quadratic Casimir implies that the constant curvature algebra, though finitely presented, is infinite dimensional.

A disadvantage of totally symmetric tensors as compared to differential forms is that in a given dimension $d \equiv n+1$, the space of symmetric tensors is unrestricted, i.e. the spectrum of $\mathbf{N}$ is $\mathbb{N} \cup\{0\}$ as compared to the $2^{n+1}$ possible differential forms ${ }^{2}$. (Of course, for physical higher spin applications, this is in fact an advantage.) Hence practical computations in general necessitate arbitrary functions (power series) in the the operators tr and $\mathbf{g}$. A calculus for such computations is provided by our next result:

The first step is to enlarge the constant curvature algebra by a certain square root of the Casimir

$$
\mathcal{T} \equiv-\sqrt{\left(\frac{n}{2}-\frac{1}{2}\right)^{2}-\mathbf{c}}
$$

and in addition we define $\mathcal{N} \equiv \mathbf{N}+\frac{n}{2}-\frac{1}{2}$. This allows us to form the operator $\mathcal{N}+\mathcal{T}$ whose eigenstates are $k$-fold trace-free tensors, namely

$$
\operatorname{tr}^{k} \varphi=0 \neq \operatorname{tr}^{k-1} \varphi \Longrightarrow(\mathcal{N}+\mathcal{T}) \varphi=2 k \varphi
$$

Then introducing

$$
\widetilde{\operatorname{div}} \equiv(\mathcal{N}-\mathcal{T}) \operatorname{div}-\operatorname{grad} \operatorname{tr}
$$

and similarly for the formal adjoint grad, the constant curvature algebra is presented by the six relations

$$
\begin{aligned}
\operatorname{tr} \mathcal{N}=(\mathcal{N}+2) \operatorname{tr}, & \operatorname{trgrad}=\widetilde{\operatorname{grad}} \frac{\mathcal{N}-\mathcal{T}+4}{\mathcal{N}-\mathcal{T}+2} \operatorname{tr}, \\
\operatorname{gtr}= & \mathcal{N}^{2}-\mathcal{T}^{2}=\operatorname{trg}-4 \mathcal{N}-4, \\
\widetilde{\operatorname{div}} \mathcal{T}=(\mathcal{T}-1) \widetilde{\operatorname{div}}, \quad & \widetilde{\operatorname{div}} \mathcal{N}=(\mathcal{N}+1) \widetilde{\operatorname{div}},
\end{aligned}
$$

\footnotetext{
${ }^{2}$ Higher spin gauge fields of mixed symmetry expressed by expanding Young tableaux in columns, rather than rows, are studied in $[3,4,5]$.
} 


$$
\begin{aligned}
\widetilde{\operatorname{div} \operatorname{grad}} & =\widetilde{\operatorname{grad}} \widetilde{\operatorname{div}} \frac{(\mathcal{N}-\mathcal{T}+2) \mathcal{T}^{2}}{(\mathcal{N}-\mathcal{T})\left(\mathcal{T}^{2}-1\right)} \\
& -2 \frac{\left(\square-\frac{(n-1)^{2}}{2}+2 \mathcal{T}^{2}\right)(\mathcal{N}-\mathcal{T}+2) \mathcal{T}^{2}}{\mathcal{N}-\mathcal{T}}
\end{aligned}
$$

and their formal adjoints where all other products are commutative. In particular, observe that the $s l(2, \mathbb{R})$ action on the pair $(\widetilde{\text { div }}, \widetilde{\text { grad }})$ is diagonal. These relations provide a calculus for constant curvature algebra computations in terms of rational functions of $(\mathcal{N}, \mathcal{T})$.

As the first, of what we anticipate to be many, applications of this calculus we present the massive higher spin action generating function on a constant curvature manifold ${ }^{3}$

$$
\begin{aligned}
& S=\varphi\left[\square+\mu^{2}-\left(\mathcal{N}+\frac{1}{2}\right)^{2}+2\left(\mathcal{N}-\frac{n}{2}+\frac{1}{2}\right)\left(\mathcal{N}+\frac{n}{2}-\frac{1}{2}\right)-\frac{1}{4}(\widetilde{\operatorname{grad}}-\mathbf{g} \widetilde{\operatorname{div}}) \frac{1}{\mathcal{N}^{2}} \widetilde{\operatorname{div}}\right] \delta_{\operatorname{tr}} \varphi \\
& +\varphi\left[\frac{4 \mathcal{T}\left(\mathcal{T}-\frac{1}{2}\right)}{(\mathcal{N}+\mathcal{T})(\mathcal{T}-1)}\left(\square+2\left[\mathcal{T}-\frac{n}{2}+\frac{1}{2}\right]\left[\mathcal{T}+\frac{n}{2}-\frac{1}{2}\right]-\frac{1}{4} \frac{\mathrm{grad}}{\mathcal{T}\left(\mathcal{T}-\frac{3}{2}\right)(\mathcal{N}-\mathcal{T}+2)} \widetilde{\mathrm{div}}\right)\right. \\
& \left.-\left(\mu^{2}-\left[\mathcal{T}+\frac{1}{2}\right]^{2}\right)(\mathcal{N}-\mathcal{T}-1)\right] \frac{\left(\frac{\mathcal{N}-\mathcal{T}+1}{2}\right)_{\frac{\mathcal{N}+\mathcal{T}-2}{2}}}{\left(\frac{1}{2}\right)_{\frac{\mathcal{N}+\mathcal{T}-2}{2}}} \Pi_{\operatorname{tr}} \varphi \\
& +\left\{2 \varphi \mathbf{g}\left[-\widetilde{\operatorname{grad}} \frac{\mu-\mathcal{T}+\frac{3}{2}}{(\mathcal{N}+\mathcal{T}+2)}+\mathrm{g} \frac{\left(\mu+\mathcal{T}-\frac{3}{2}\right)(\mathcal{N}-\mathcal{T}+5)}{(\mathcal{N}-\mathcal{T}+2)(\mathcal{N}+\mathcal{T}+1)(\mathcal{N}+\mathcal{T}+4)} \widetilde{\mathrm{div}}\right]\right. \\
& +\chi\left[\frac{4 \mathcal{T}\left(\mathcal{T}-\frac{1}{2}\right)}{\mathcal{T}-1}\left(\square+2\left[\mathcal{T}-\frac{n}{2}+\frac{1}{2}\right]\left[\mathcal{T}+\frac{n}{2}-\frac{1}{2}\right]-\frac{1}{4} \widetilde{\operatorname{grad}} \frac{\mathcal{T}-1}{\mathcal{T}\left(\mathcal{T}-\frac{3}{2}\right)(\mathcal{N}-\mathcal{T}+2)} \widetilde{\mathrm{div}}\right)\right. \\
& \left.\left.-\left(\mu^{2}-\left[\mathcal{T}+\frac{1}{2}\right]^{2}\right)(\mathcal{N}-\mathcal{T}+2)(\mathcal{N}+\mathcal{T}+3)\right] \frac{\mathcal{N}-\mathcal{T}+2}{\mathcal{N}+\mathcal{T}+1}\right\} \frac{\left(\frac{\mathcal{N}-\mathcal{T}+5}{2}\right)}{\left(\frac{1}{2}\right)_{\frac{\mathcal{N}+\mathcal{T}}{2}}} \chi .
\end{aligned}
$$

Specializing to $(\mathbf{N}-s) \varphi=(\mathbf{N}-s+3) \chi$, this action describes a spin $s$ field of mass $m^{2}=-\mu^{2}+\left(s+\frac{n}{2}-2\right)^{2}$ in terms of a pair of unconstrained fields $(\varphi, \chi)$. Massless and partially massless theories $[7,8,9,10,11,12]$ appear at tuned values of $m^{2}$ for which this action enjoys gauge invariances. A complete explanation may be found in Section 6. Also, in the flat space limit, this result provides a generating function for the original massive higher spin actions of [6].

The remainder of the Article is organized thus; in Section 2 we define and describe the constant curvature algebra in detail. These results are new.

\footnotetext{
${ }^{3}$ Here $(x)_{n}$ is the Pochhammer symbol and the projector $\Pi_{\mathbf{t r}}=1-\delta_{\mathbf{t r}}=$ $\mathrm{g} \frac{1}{(\mathcal{N}+2)^{2}-\mathcal{T}^{2}}$ tr.
} 
The following Section 3 applies this algebra to the massless action for higher spins in constant curvature backgrounds, a subject studied already in detail in $[13,14]$. In Section 4 we summarize the radial reduction technique for reducing from Minkowski to constant curvature theories developed in [15]. The combination of this reduction technique and our constant curvature algebra allows us to solve the problem of writing a generating function for massive, constant curvature theories of arbitrary higher spins in Section 5 . Some results exist already for these theories ${ }^{4}$ [17], but our algebraic generating function formalism, is far more compact and suitable for generalizations, including perhaps interactions. In Section 6, we analyze partially massless gauge symmetries of the massive action in terms of degeneracies of the flat, massless gauge transformations in one dimension higher. This result is also new. The fermionic version of the constant curvature algebra appears in Section 7 in terms of the corresponding superalgebra. We also write massless constant curvature higher spin actions in terms of this algebra. Our reformulation of all higher spins in terms of a single scalar field living on the total space of the cotangent bundle $T^{*} M$ is given in Section 8. Our conclusions are in Section 9. In the Appendices, various aspects tangential to the main developments are given. These include a new on-shell formulation of partially massless higher spins in terms of residual gauge transformations, as well as further details on the constant curvature algebra, in particular its interpretation in terms of a non-commutative harmonic oscillator.

\section{Constant Curvature Algebra}

Let $M$ be an $n+1$ dimensional constant curvature manifold. Its Riemann curvature is

$$
R_{\mu \nu \rho \sigma}=-\frac{2 \Lambda}{n} g_{\mu[\rho} g_{\sigma] \nu}
$$

where the parameter $\Lambda$ is the cosmological constant, positive in de Sitter and negative in Anti de Sitter space. Throughout we will denote $n \equiv d-1$ and work in units $^{5} \Lambda=n$. The actions of commutators of covariant derivatives

\footnotetext{
${ }^{4}$ A light cone formulation of totally symmetric Anti de Sitter higher spins was given in $[16]$.

${ }^{5}$ This choice is germane to de Sitter space, however, upon reinstating $\Lambda$ by dimensional analysis, all our formulæapply to Anti de Sitter as well.
} 
are therefore summarized by the vector-spinor example ${ }^{6}$

$$
\left[D_{\mu}, D_{\nu}\right] \psi_{\rho}=2 g_{\rho[\mu} \psi_{\nu]}+\frac{1}{2} \gamma_{\mu \nu} \psi_{\rho}
$$

Let $\odot T^{*} M$ be the symmetric tensor bundle whose sections are expressed in terms of tensor fields

$$
\odot T^{*} M \ni \Phi=\varphi_{\mu_{1} \ldots \mu_{s}} d x^{\mu_{1}} \ldots d x^{\mu_{s}}
$$

Note, there is no restriction to elements of definite index content, so sums and products of elements with differing values of $s$ are allowed. Here the product of differentials $d x^{\mu}$ stands for the symmetric tensor product ${ }^{7}$

$$
d x^{\mu_{1}} \ldots d x^{\mu_{s}} \equiv \frac{1}{s !} \sum_{\sigma} d x^{\mu_{\sigma(1)}} \otimes \cdots \otimes d x^{\mu_{\sigma(s)}} .
$$

In this way, we can regard symmetric tensors simply as functions of the commuting differential $d x^{\mu}$. We define various operations on symmetric tensors ${ }^{8}$. Firstly

$$
\partial_{\mu}: \odot T^{*} M \rightarrow \odot T^{*} M
$$

where

$$
\partial_{\mu} d x^{\nu}=\delta_{\mu}^{\nu}+d x^{\nu} \partial_{\mu}
$$

The coordinate vector $\partial_{\mu}$ returns zero when acting all the way to the right and obeys the Leibnitz rule on products of differentials. For example,

$$
\partial_{\mu} d x^{\nu} d x^{\rho}=2 \delta_{\mu}^{(\nu} d x^{\rho)}
$$

Importantly, the operation $\partial_{\mu}$ is fiberwise and does not act on the field-valued coefficients $\varphi_{\mu_{1} \ldots \mu_{s}}$. The other operator we need is the covariant derivative

$$
D_{\mu}: \odot T^{*} M \rightarrow \odot T^{*} M
$$

\footnotetext{
${ }^{6}$ Our metric is "mostly plus", Dirac matrices are "mostly hermitean" and the Dirac conjugate is $\bar{\psi} \equiv \psi^{\dagger} i \gamma^{0}$. We denote (anti)symmetrization with unit weight by round (resp. square) brackets. Antisymmetrized products of Dirac matrices are given by $\gamma^{\mu_{1} \ldots \mu_{n}} \equiv$ $\gamma^{\left[\mu_{1} \ldots \gamma^{\mu_{n}}\right]}$.

${ }^{7}$ Or more correctly, the Cartan product, see [18].

${ }^{8}$ This notational device has been considered before in [19]. In that notation the vector $a^{\mu}$ should be regarded as a coordinate differential $d x^{\mu}$.
} 
which acts only on the fields, not differentials

$$
D_{\mu}\left[\varphi_{\mu_{1} \ldots \mu_{s}} d x^{\mu_{1}} \ldots d x^{\mu_{s}}\right]=D_{\mu} \varphi_{\mu_{1} \ldots \mu_{s}} d x^{\mu_{1}} \ldots d x^{\mu_{s}}
$$

To achieve a completely index free notation we define a slew of additional operators built from the above ingredients:

- Index:

$$
\mathbf{N} \equiv d x^{\mu} \partial_{\mu}
$$

This operator counts indices. Its eigenvalues are simply the number of indices and eigenspaces are tensors of definite index type. For example,

$$
\mathbf{N} d x^{\mu_{1}} \ldots d x^{\mu_{s}}=s d x^{\mu_{1}} \ldots d x^{\mu_{s}} .
$$

- Trace:

$$
\operatorname{tr} \equiv g^{\mu \nu} \partial_{\mu} \partial_{\nu}
$$

This is the operation of tracing over a single pair of indices. For example,

$$
\operatorname{tr} \varphi_{\mu_{1} \ldots \mu_{s}} d x^{\mu_{1}} \ldots d x^{\mu_{s}}=s(s-1) \varphi^{\mu}{ }_{\mu \mu_{3} \ldots \mu_{s}} d x^{\mu_{3}} \ldots d x^{\mu_{s}} .
$$

The kernel of this operator is the set of trace-free tensors and eigenvectors of $\mathbf{N}$ with eigenvalue 0,1 (i.e. scalar and vector fields).

- Metric:

$$
\mathbf{g} \equiv g_{\mu \nu} d x^{\mu} d x^{\nu}
$$

Forms the new tensor obtained by multiplying by the metric and symmetrizing,

$$
\text { g } \varphi_{\mu_{1} \ldots \mu_{s}} d x^{\mu_{1}} \ldots d x^{\mu_{s}}=g_{\mu_{1} \mu_{2}} \varphi_{\mu_{3} \ldots \mu_{s+2}} d x^{\mu_{1}} \ldots d x^{\mu_{s+2}} .
$$

- Divergence:

$$
\operatorname{div} \equiv \partial_{\mu} D^{\mu}
$$

As suggested by its name

$$
\operatorname{div} \varphi_{\mu_{1} \ldots \mu_{s}} d x^{\mu_{1}} \ldots d x^{\mu_{s}}=s D_{\mu} \varphi^{\mu}{ }_{\mu_{2} \ldots \mu_{s}} d x^{\mu_{1}} \ldots d x^{\mu_{s}} .
$$

The kernel of $\mathbf{d i v}$ is the same as $\mathbf{N}$, i.e. scalar fields. 
- Gradient:

$$
\operatorname{grad} \equiv d x^{\mu} D_{\mu} .
$$

This is the usual gradient operation

$$
\operatorname{grad} \varphi_{\mu_{1} \ldots \mu_{s}} d x^{\mu_{1}} \ldots d x^{\mu_{s}}=D_{\mu_{1}} \varphi_{\mu_{2} \ldots \mu_{s+1}} d x^{\mu_{1}} \ldots d x^{\mu_{s+1}} .
$$

- Laplacian:

$$
\Delta \equiv D^{\mu} D_{\mu} .
$$

An important relation in constant curvature spaces is

$$
\Delta=\operatorname{div} \operatorname{grad}-\operatorname{grad} \operatorname{div}+\mathbf{N}(\mathbf{N}+n-1)-\operatorname{gtr} .
$$

The Laplacian is related to the commutator, rather than anticommutator, of the divergence and gradient appearing in the theory of differential forms, because we work with symmetric tensors. Moreover, in the flat space limit the Laplacian coincides with the commutator of the divergence and gradient.

\subsection{Algebra of Operations}

Much mileage is gained by computing the algebra of the operators $\mathbf{g}$, grad, $\mathbf{N}$, div and tr. This is a simple calculation whose results are simplified by noting the following

1. The operator

$$
\mathbf{c}=\mathbf{g} \operatorname{tr}-\mathbf{N}(\mathbf{N}+n-1)
$$

commutes with $\mathbf{g}, \mathbf{t r}$ and $\mathbf{N}$. In fact it is the quadratic Casimir of an $s l(2)$ Lie algebra formed by this triplet.

2. The differential operator

$$
\square=\Delta+\mathbf{c}=[\operatorname{div}, \operatorname{grad}]+2 \mathbf{c},
$$

is central and is precisely the wave operator introduced some time ago by Lichnerowicz [2]. 
Without further ado, we present the algebra of operators on symmetric tensors

$$
\begin{gathered}
{[\mathbf{t r}, \mathbf{g}]=4 \mathbf{N}+2 n+2, \quad[\mathbf{t r}, \operatorname{grad}]=2 \operatorname{div}, \quad[\operatorname{div}, \mathbf{g}]=2 \operatorname{grad}} \\
{[\operatorname{div}, \operatorname{grad}]=\square-2 \mathbf{c} .}
\end{gathered}
$$

Commutators with the index operator $\mathbf{N}$ are all of the form $[\mathbf{N}, \mathcal{O}]=\operatorname{wt}_{\mathcal{O}} \cdot \mathcal{O}$ where the weights are tabulated below

$$
\begin{array}{c|cccccc}
\mathcal{O} & \text { tr } & \text { div } & \mathbf{N} & \square & \text { grad } & \text { g } \\
\hline \text { wt }_{\mathcal{O}} & -2 & -1 & 0 & 0 & 1 & 2
\end{array}
$$

All other commutators vanish and the algebra is neatly presented by the diagram in Figure 1. We will refer to this algebra as the "constant curvature algebra" and its flat space limit as the "flat space algebra". Finally, it is important to note that although the flat space algebra is a Lie algebra, its constant curvature deformation by the Casimir c, living in the universal enveloping algebra of $s l(2, \mathbb{R})$, is not. The operators div and grad do not commute with $\mathbf{c}$ but rather higher commutators produce an infinite sequence of additional operators. We stress that the consistency of the algebra is, however, guaranteed thanks to its explicit representation acting on symmetric tensors.

\subsection{Analysis of the Operator Algebra}

Our first remark is that $\mathbf{t r}=-2 e_{+}, \mathbf{g}=2 e_{-}$and $\mathbf{N}=-h-n / 2-1 / 2$ generate an $\operatorname{sl}(2, \mathbb{R})$ Lie algebra

$$
\left[h, e_{ \pm}\right]= \pm 2 e_{ \pm}, \quad\left[e_{+}, e_{-}\right]=h .
$$

The quadratic Casimir $\mathbf{c}=-h^{2}-2\left\{e_{+}, e_{-}\right\}+\frac{1}{4}(n+1)(n-3)$ labels irreducible representations. Note that there is a natural adjoint operation

$$
\operatorname{tr}^{\dagger}=\mathbf{g}, \quad \operatorname{div}^{\dagger}=-\operatorname{grad}, \quad N^{\dagger}=N, \quad \square^{\dagger}=\square .
$$

Therefore, unitary $s l(2)$ irreducible representations are highest weight where the highest weight state $\Phi$ obeys

$$
\mathbf{N} \Phi=s \Phi, \quad \operatorname{tr} \Phi=0
$$




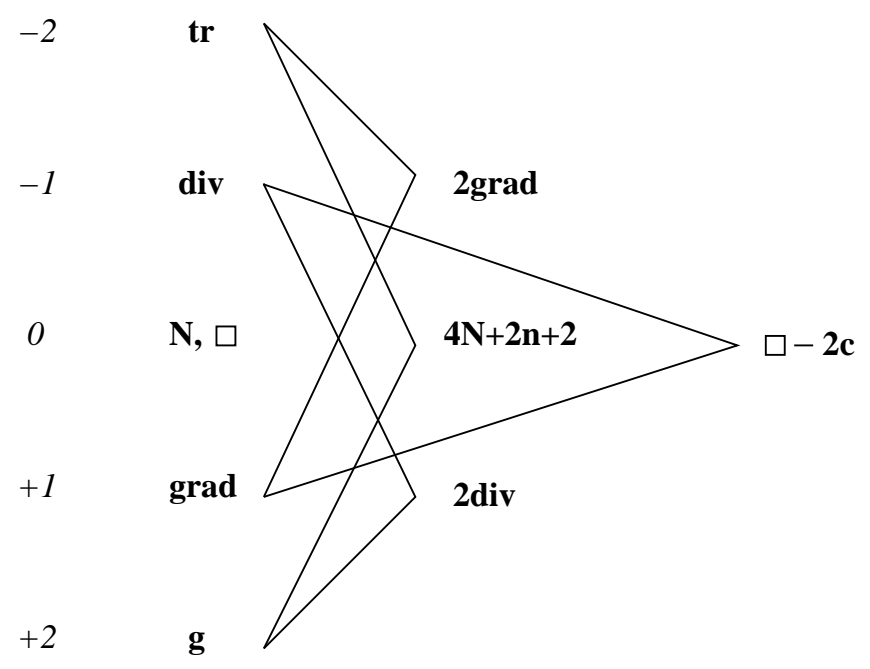

Figure 1: The algebra of operators on symmetric tensors living on $n+1$ dimensional constant curvature manifolds. The left-hand column enumerates the weight with respect to the index operator $\mathbf{N}$. The two rightmost columns are the results for non-trivial/vanishing commutators. 
i.e. an $s$-index traceless symmetric tensor. The representation is spanned by states $\left\{\Phi, \mathbf{g} \Phi, \mathbf{g}^{2} \Phi, \ldots\right\}$ with $N$-eigenvalues $s, s+2, s+4, \ldots$. This representation is infinite dimensional, as must be on general grounds, and all states are eigenvectors of the Casimir $\mathbf{c}$ with eigenvalue $-s(s+n-1)$. Indeed, it is the well-known discrete series representation of $\operatorname{sl}(2, \mathbb{R})$.

Examining Figure 1, we observe that the doublet $\{$ div, grad $\}$ is simply the fundamental representation of $\operatorname{sl}(2, \mathbb{R})$. Regarding the doublet as coordinates for the plane $\mathbb{R}^{2}$, the triplet of operators $\{\mathbf{t r}, \mathbf{N}, \mathbf{g}\}$ act as infinitesimal generators of unimodular changes of basis. However, since div and grad fail to commute, what we really have is a non-commutative plane. In flat space, we have only the central deformation ${ }^{9} \square$ (on dimensional grounds the coefficient of $-2 c$ in (33) is $\Lambda / n \rightarrow 0$ ), while for constant curvature manifolds there is an additional deformation by the Casimir $\mathbf{c}$ which is central in the $s l(2, \mathbb{R})$ subalgebra only. Finally, note that setting $\square-2 c=0$, the algebra is a Lie algebra, closed under commutation and is simply the $(2,1)$-parabolic of $\operatorname{sl}(3, \mathbb{R})$ modded out by its connected center.

There is an an interesting relationship between the constant curvature algebra and the harmonic oscillator. Also, the constant curvature algebra in $n+1$ dimensions can be embedded in its flat space counterpart in one dimension higher. Since these aspects are tangential to our main story, they are presented in Appendix A. The computations we encounter in this Article involve functions of arbitrarily high powers of the operators $\mathbf{g}$ and $\mathbf{t r}$ (although typically the overall weight $N$ remains small). There are two approaches to handle this difficulty. The first is choose a standard ordering, for example normal ordering with $\mathbf{g}$ 's to the left and tr's to the right. Although this method will be useful at times, the second method is far more powerful: namely to maximally commute operators $\mathbf{g}$ and $\mathbf{t r}$ so that they are adjacent and then relate their product $\mathbf{g} \operatorname{tr}$ to the Casimir $\mathbf{c}$. We detail these two techniques in the following Sections.

\subsubsection{Normal Ordering and Hadamard Products}

For later use, we define a normal ordering for the constant curvature and flat space algebras. Our convention is to order operators with respect to their index operator $\mathbf{N}$ weights, i.e.

$$
\mathrm{g}>\operatorname{grad}>\mathbf{N}, \square>\operatorname{div}>\operatorname{tr} .
$$

\footnotetext{
${ }^{9}$ In flat space, $\square$ becomes the usual d'Alembertian.
} 
We denote normal ordering as usual by : ... :, so for example,

$$
\operatorname{trg}=: \mathbf{g} \operatorname{tr}:+4 \mathbf{N}+2 n+2 \text {. }
$$

In addition, for later reference we will also need to compute normal ordered expressions of the following type ${ }^{10}$ :

$$
f(\partial \sqrt{\mathbf{g}}): G(\mathbf{g}, \operatorname{grad}, \mathbf{N}, \mathbf{d i v}, \mathbf{t r}): g(d u \sqrt{\mathbf{t r}}) .
$$

Here $[\partial, d u]=1$ and $\partial$ vanishes when pushed all the way to the right (as does $d u$ to the left). To solve this problem, note that only terms with equal numbers of $\partial$ and $d u$ 's contribute so if $f(x)=\sum_{n} f_{n} x^{n}$ and $g(x)=\sum_{n} g_{n} x^{n}$ we obtain

$$
f(\partial \sqrt{\mathbf{g}}): G: g(d u \sqrt{\mathbf{t r}})=:(f * g)(\sqrt{\mathbf{g} \mathbf{t r}}) G:,
$$

where the generalized Hadamard product

$$
(f * g)(x) \equiv \sum_{n} n ! f_{n} g_{n} x^{n} .
$$

\subsubsection{Casimir Basis}

For many applications, we deal with operators involving high powers of $\mathbf{g}$ and tr. These can be handled by the normal ordering prescription given above. A more potent technique is to express the product $\mathbf{g}$ tr in terms of the Casimir $\mathbf{c}$ and $\mathbf{N}$ so that one deals simply with functions thereof:

To begin with, introduce operators

$$
\begin{aligned}
\mathcal{N} & \equiv \mathbf{N}+\frac{n}{2}-\frac{1}{2} . \\
\mathcal{T} & \equiv-\sqrt{\frac{(n-1)^{2}}{4}-\mathbf{c} .}
\end{aligned}
$$

Here we are enlarging the constant curvature algebra by the above square root of the $\operatorname{sl}(2)$ Casimir. In fact, we will even employ rational functions of the operators $\mathcal{N}, \mathcal{T}$. This maneuver is extremely useful because the combination $\mathcal{N}+\mathcal{T}$ measures how many traces annihilate a symmetric tensor

$$
(\mathcal{N}+\mathcal{T}) \mathbf{g}^{k} \xi=2 k \mathbf{g}^{k} \xi \text { when } \operatorname{tr} \xi=0
$$

\footnotetext{
${ }^{10}$ Note that typically $f$ and $g$ will be even functions so that no fractional powers of the operators $\mathbf{g}$ and $\mathbf{t r}$ appear.
} 
Note also that

$$
\operatorname{g} \operatorname{tr}=(\mathcal{N}+\mathcal{T})(\mathcal{N}-\mathcal{T})=\operatorname{tr} \mathbf{g}-4 \mathcal{N}-4
$$

and $\mathcal{T}$ commutes with $\mathbf{g}, \mathbf{N}$ and $\operatorname{tr}$ while $\mathbf{c}=-\left(\mathcal{T}+\frac{n}{2}-\frac{1}{2}\right)\left(\mathcal{T}-\frac{n}{2}+\frac{1}{2}\right)$. Hence

$$
:(\mathbf{g} \mathbf{t r})^{k}:=\mathbf{g}^{k} \mathbf{t r}^{k}=4^{k}\left(-\frac{\mathcal{N}+\mathcal{T}}{2}\right)_{k}\left(\frac{\mathcal{T}-\mathcal{N}}{2}\right)_{k}
$$

where the Pochhammer symbol $(x)_{k} \equiv x(x+1) \cdots(x+k-1)$. This observation allows us to convert normal ordered expressions to ones involving the Casimir and Index operators only. The archetypal example is

$$
: \cosh \sqrt{\mathbf{g} \operatorname{tr}}:=\sum \frac{\mathbf{g}^{k} \mathbf{t r}^{k}}{(2 k) !}=\frac{\left(\frac{1+\mathcal{N}-\mathcal{T}}{2}\right)_{\frac{\mathcal{N}+\mathcal{T}}{2}}}{\left(\frac{1}{2}\right)_{\frac{\mathcal{N}+\mathcal{T}}{2}}}
$$

where we note that the eigenvalues of $\frac{\mathcal{N}+\mathcal{T}}{2}$ are always non-negative integers.

\section{Casimir Algebra}

It is most useful to introduce trace and spin modifications of the operators div and grad

$$
\widetilde{\operatorname{div}} \equiv(\mathcal{N}-\mathcal{T}) \operatorname{div}-\operatorname{grad} t r, \quad \widetilde{\operatorname{grad}} \equiv \operatorname{grad}(\mathcal{N}-\mathcal{T})-\operatorname{gdiv}
$$

which have simple commutation relations with the operator $\mathcal{T}$

$$
\left[\mathcal{T},\left(\frac{\widetilde{\mathrm{div}}}{\operatorname{grad}}\right)\right]=\left(\frac{\widetilde{\mathrm{div}}}{-\widetilde{\operatorname{grad}}}\right) .
$$

We may invert the relations (48) by solving a $2 \times 2$ operator-valued matrix problem and find

$$
\begin{aligned}
\operatorname{div} & =-\frac{1}{2}\left(\frac{1}{\mathcal{T}} \widetilde{\operatorname{div}}+\widetilde{\operatorname{grad}} \operatorname{tr} \frac{1}{\mathcal{T}(\mathcal{N}-\mathcal{T})}\right) \\
\operatorname{grad} & =-\frac{1}{2}\left(\widetilde{\operatorname{grad}} \frac{1}{\mathcal{T}}+\frac{1}{\mathcal{T}(\mathcal{N}-\mathcal{T})} \operatorname{g\operatorname {div}}\right)
\end{aligned}
$$

Using these relations it is now not difficult to reproduce the algebra (8) quoted in the Introduction. 
Let us emphasize what we have achieved: The infinite dimensional Lie algebra (33) has been rephrased as a finitely presented algebra in seven generators. Needless to say the Casimir basis is highly advantageous for calculations.

We end this Section with a warning and two new operators: Computations involving rational functions of $\mathcal{N}$ and $\mathcal{T}$ risk encountering singular denominators. The most important example is the operator

$$
\boldsymbol{\Pi}_{\mathrm{tr}} \equiv \mathbf{g} \frac{1}{(\mathcal{N}+2)^{2}-\mathcal{T}} \operatorname{tr} .
$$

One might think that this operator were unity by commuting the denominator left past $\mathbf{g}$ (which shifts $\mathcal{N}$ by -2 ) and then using the relation $\mathrm{g} \operatorname{tr}=\mathcal{N}^{2}-\mathcal{T}^{2}$ to conclude that $\left(\mathcal{N}^{2}-\mathcal{T}^{2}\right)^{-1} \mathbf{g} \operatorname{tr}=1$. This is clearly false. The operator $\Pi_{\operatorname{tr}}$ is actually a projector, returning zero on traceless tensors and unity otherwise. We also define its complement $\delta_{\mathbf{t r}} \equiv 1-\boldsymbol{\Pi}_{\mathbf{t r}}$ which projects onto traceless tensors.

\section{Generating Function for Massless Higher Spin Actions}

Our first physics application of the constant curvature and flat space algebras is to write a generating function for massless higher spins. The massless theories are very well known and have rather simple actions, indeed our result amounts to little more than simply replacing the eigenvalue $s$ of the index operator by the operator $\mathbf{N}$. The key point, however, is that we present an action depending on fields whose spin need not not be specified (and can even be indefinite) and which generates all massless actions when evaluated on fields of definite spins.

The minimal covariant field content for massless higher spins is a doubly traceless field $\varphi$ :

$$
\operatorname{tr}^{2} \varphi=0 .
$$

The correct physical degrees of freedom are ensured by the gauge invariance

$$
\delta \varphi=\operatorname{grad} \xi,
$$

where the gauge parameter $\xi$ is tracefree:

$$
\operatorname{tr} \xi=0 .
$$


To study actions we need to introduce an inner product on the space of symmetric tensor fields

$$
\langle\varphi, \chi\rangle=\int \sqrt{-g} \varphi^{\dagger} \chi
$$

The adjoint operation $\dagger$ on operators was given in (36) and acts on fields via

$$
\left[\varphi_{\mu_{1} \ldots \mu_{s}}^{*} d x^{\mu_{1}} \cdots d x^{\mu_{s}}\right]^{\dagger}=\varphi^{\mu_{1} \ldots \mu_{s}} \partial_{\mu_{1}} \cdots \partial_{\mu_{s}} .
$$

The operators $\partial_{\mu_{i}}$ must then be passed all the way to the right where they return zero. It is extremely important to note that this dualization is metric dependent since $\varphi^{\mu_{1} \ldots \mu_{s}}=g^{\mu_{1} \nu_{1}} \cdots g^{\mu_{s} \nu_{s}} \varphi_{\nu_{1} \ldots \nu_{s}}$. We will often use the shorthand notation $\langle\varphi, \chi\rangle \equiv \int \varphi \chi$, so for example when $\varphi=\varphi_{\mu} d x^{\mu}$ and $\chi=\chi_{\mu} d x^{\mu}$ we have

$$
\int \varphi \chi=\int \sqrt{-g} \varphi_{\mu}^{*} \chi^{\mu}
$$

The action for massless fields is then uniquely determined (assuming quadratic derivatives) by the gauge invariance (53) and reads ${ }^{11}$

$$
\begin{gathered}
S=\int \varphi\left\{\square+2(\mathbf{N}-1)(\mathbf{N}+n-2)-\operatorname{grad} \operatorname{div}+\frac{1}{2}\left[\operatorname{grad}^{2} \mathbf{t r}+\mathbf{g d i v}^{2}\right]\right. \\
\left.-\frac{1}{2} \mathbf{g}\left[\square+2 \mathbf{N}(\mathbf{N}+n)+n+\frac{1}{2} \operatorname{grad} \operatorname{div}\right] \mathbf{t r}\right\} \varphi \equiv \int \varphi G \varphi .
\end{gathered}
$$

The half integer spin generalization of this result is given in Section 7.1. Its voracity is easily verified by computing the Bianchi Identity

$$
\operatorname{div} G=\mathbf{g} X \equiv 0 \bmod \mathbf{g},
$$

corresponding to the gauge invariance (53).

\footnotetext{
${ }^{11}$ Integrating by parts, we may also write

$S=-\int\left\{|\operatorname{grad} \varphi|^{2}-2|\operatorname{div} \varphi|^{2}+(\operatorname{div} \varphi)^{\dagger} \operatorname{grad} \operatorname{tr} \varphi-\frac{1}{2}|\operatorname{grad} \operatorname{tr} \varphi|^{2}+\frac{1}{4}|\operatorname{div} \operatorname{tr} \varphi|^{2}\right.$$$
\left.-2 \varphi^{\dagger}(\mathbf{N}-1)(\mathbf{N}+n-2) \varphi+\frac{1}{2}(\operatorname{tr} \varphi)^{\dagger}(2 \mathbf{N}(\mathbf{N}+n)+n) \operatorname{tr} \varphi\right\} .
$$ 


\section{Radial Dimensional Reduction}

The $d \equiv n+1$ dimensional de Sitter spacetime ${ }^{12}$ is most simply described by pulling back the flat metric

$$
d s^{2}=-d Z^{2}+\sum_{i=1}^{d}\left(d X^{i}\right)^{2}
$$

in $d+1$ dimensions to the hyperboloid

$$
-Z^{2}+\sum_{i=1}^{d}\left(X^{i}\right)^{2}=\frac{n}{\Lambda} .
$$

As usual, we set $\Lambda / n=1$, factors of which can be readily reinstated by examining dimensions. In particular, choosing new Minkowski coordinates $Z=e^{u} \xi^{0}, X^{i}=e^{u} \xi^{i}$ where $\xi^{M} \eta_{M N} \xi^{N}=1$ so that $e^{2 u}=-Z^{2}+\sum_{i=1}^{d}\left(X^{i}\right)^{2}$, we have $d s^{2}=e^{2 u}\left(d u^{2}+d \xi^{M} \eta_{M N} d \xi^{N}\right)$ and in turn

$$
d s^{2}=\exp (2 u)\left(d u^{2}+d s_{d S}^{2}(x)\right)
$$

The de Sitter metric $d s_{d S}^{2}(x)$ in the second line appears upon choosing a parametrization of the hyperboloid $\xi^{M}=\xi^{M}\left(x^{\mu}\right)$. In particular observe that the operator $\partial_{u} \equiv \frac{\partial}{\partial u}$ is a homothetic Killing vector $\left[\partial_{u}, d s^{2}\right]=2 d s^{2}$. For this reason, a (rather elegant) radial dimensional reduction from flat to de Sitter space with respect to the conformal isometry generated by $\partial_{u}$ was suggested in $[15]$.

This reduction is easily understood by looking at the simplest example of a massless scalar

$$
S=-\frac{1}{2} \int d Z d^{d} X \partial_{M} \Phi \eta^{M N} \partial_{N} \Phi
$$

In the coordinates $\left(u, x^{\mu}\right)$ the action is

$$
S=-\frac{1}{2} \int d u e^{n u} d^{d} x \sqrt{-g_{d S}}\left\{\partial_{\mu} \Phi g_{d S}^{\mu \nu} \partial_{\nu} \Phi+\left[\partial_{u} \Phi\right]^{2}\right\} .
$$

\footnotetext{
${ }^{12}$ All computations in this Section generalize trivially to Anti de Sitter (see [15] for details). We concentrate on de Sitter for definiteness only.
} 
Crucially, we wish to make a Scherk-Schwarz reduction [20] with respect to the log-radial coordinate $u \in(-\infty, \infty)$. Therefore we demand that the measure of the action functional be simply $d u$ which requires the Weyl rescaling

$$
\Phi=e^{-n u / 2} \varphi
$$

We remark, that $n / 2$ is of course the scaling dimension of the field $\Phi$. The action now becomes

$$
S=-\frac{1}{2} \int d u d^{d} x \sqrt{-g_{d S}}\left\{\partial_{\mu} \varphi g_{d S}^{\mu \nu} \partial_{\nu} \varphi+\left[\left(\partial_{u}-\frac{n}{2}\right) \varphi\right]^{2}\right\} .
$$

We may now Scherk-Schwarz reduce to the sector of fixed log-radial momentum $^{13}$

$$
\partial_{u}=i m
$$

The reduced action reads

$$
S_{d S}=-\frac{1}{2} \int d^{d} x \sqrt{-g_{d S}}\left\{\partial_{\mu} \varphi g_{d S}^{\mu \nu} \partial_{\nu} \varphi+\left[m^{2}+\frac{n^{2}}{4}\right] \varphi^{2}\right\}
$$

A simple consistency check verifies that the reduced equation of motion $\left(-\square_{d S}+m^{2}+\frac{n \Lambda}{4}\right) \varphi=0$ obtained by varying the reduced action (68) coincides with original $d+1$ dimensional field equation $-\square_{d+1} \Phi=0$ in this fixed momentum sector. In the next Section we apply the radial dimensional reduction technique to derive a generating function for all massive and partially massless theories in de Sitter space.

\section{Generating Functions for Massive Actions}

An important observation is that constant curvature massive and partially massless systems in dimension $d$ are really just subsectors of the $d+1$ dimensional, flat massless theory. In particular as we shall see, the partially massless theories arise as degeneracies of the $d+1$ dimensional gauge variations. It was first observed that massive higher spins could be obtained by dimensional reductions in [21] (see [22] for more recent developments).

\footnotetext{
${ }^{13}$ It is most important to note that $\int d u f(u) g(u)=\frac{1}{2 \pi} \int d p_{u} f\left(-p_{u}\right) g\left(p_{u}\right)$ reflecting logradial momentum conservation.
} 
Our starting point therefore is the massless higher spin system in $d+1$ flat dimensions formulated in terms of a doubly traceless field $\Phi$,

$$
\operatorname{Tr}^{2} \Phi=0
$$

subject to a gauge invariance

$$
\delta \Phi=\operatorname{Grad} \Xi
$$

with traceless gauge parameter

$$
\operatorname{Tr} \Xi=0 .
$$

We distinguish $d+1$ dimensional flat operators from their $d$ dimensional constant curvature counterparts by capitalization.

The first maneuver is to make Weyl rescalings mimicking the scalar case (65) with the usual spin dependence of the conformal weight ${ }^{14}$ :

$$
\Phi \equiv e^{\left(\mathbf{N}_{d+1}-n / 2\right) u} \phi, \quad \Xi \equiv e^{\left(\mathbf{N}_{d+1}-n / 2+1\right) u} \xi .
$$

Next we need to decompose the $d+1$ tensorial structure of $\Phi$ and $\Xi$ into $d$ dimensional tensors. Both the field $\phi$ and gauge parameter $\xi$ can be expanded in a power series in the commuting differential $d u$

$$
\phi=\varphi_{0}+d u \varphi_{1}+\frac{1}{2 !} d u^{2} \varphi_{2}+\frac{1}{3 !} d u^{3} \varphi_{3}+\cdots, \quad \xi=\xi_{1}+d u \xi_{2}+\cdots .
$$

If $\phi$ is of definite index, i.e. $\mathbf{N}_{d+1} \phi=s \phi$, the fields $\varphi_{t}$ have definite index in one dimension lower $\mathbf{N} \varphi_{t}=(s-t) \varphi_{t}$. In turn the gauge parameters $\xi_{t}$ then have index $\mathbf{N} \xi_{t}=(s-t) \xi_{t}$.

A beauty of the dimensional reduction technique is that the towers of auxiliary fields required for massive covariant actions appear naturally. Moreover, in our approach, these towers of auxiliaries are packaged in a minimal set of unconstrained covariant fields.

To solve the traceless and double traceless conditions (71) and (69) in terms of a minimal set of unconstrained $d$ dimensional fields, observe that

\footnotetext{
${ }^{14}$ Note that although the appearance of $\mathbf{N}_{d+1}$ in the scaling dimensions is canonical, it can be deduced by looking at the leading behavior of the flat d'Alembertian $\square_{d+1}=$ $\partial_{u}^{2}+[s+n / 2] \partial_{u}+\cdots$ acting on a spin $s$ field. The Weyl rescalings quoted above precisely cancel the terms linear in $\partial_{u}$.
} 
$[\partial, \operatorname{tr}]=0$ so $\operatorname{Tr} \Xi=0 \Rightarrow\left(\partial^{2}+\operatorname{tr}\right) \xi=0$ is the equation for a harmonic oscillator with solution

$$
\xi=\cos (d u \sqrt{\mathbf{t r}}) \xi_{1}+\frac{\sin (d u \sqrt{\mathbf{t r}})}{\sqrt{\mathbf{t r}}} \xi_{2} .
$$

We have chosen boundary conditions such that $\xi$ is determined in terms of the $d$ dimensional unconstrained gauge parameters $\xi_{1}$ and $\xi_{2}$. We can solve the double trace condition (69) in exactly the same way and find

$$
\phi=\cos (d u \sqrt{\mathbf{t r}})\left[\varphi_{0}-\frac{1}{2} d u\left(\varphi_{1}+\frac{1}{\operatorname{tr}} \varphi_{3}\right)\right]+\frac{\sin (d u \sqrt{\operatorname{tr}})}{2 \sqrt{\operatorname{tr}}}\left[3 \varphi_{1}+\frac{1}{\operatorname{tr}} \varphi_{3}+d u\left(\operatorname{tr} \varphi_{0}+\varphi_{2}\right)\right] .
$$

Note that despite the appearance of explicit inverse powers of $\mathbf{t r}$, the above expressions are actually regular at $\mathbf{t r}=0$ and at any fixed spin their expansions terminate. As boundary conditions we have taken the leading terms of the expansions displayed in (73). In particular this means that a massive spin $s$ field is described by four unconstrained fields $\varphi_{0,1,2,3}$ of spin $s, s-1, s-2, s-3$ modulo Stückelberg gauge transformations parameterized by two unconstrained fields $\xi_{1}$ and $\xi_{2}$ with spins ${ }^{15} s-1, s-2$.

\subsection{Radially Reduced Gauge Variations}

To compute the radially reduced gauge transformations of the four independent, unconstrained, $d$ dimensional fields $\varphi_{0,1,2,3}$ we first need to radially reduce the operator Grad. This is a simple matter of spelling out the Christoffel symbols for the flat metric in log-radial coordinate (62)s and substituting these in Grad $=d u D_{u}+d x^{\mu} D_{\mu}$ acting on an arbitrary tensor field. This easy, but slightly tedious computation yields

$$
\operatorname{Grad}=\operatorname{grad}+\mathbf{g} \partial+d u\left(\partial_{u}-2 N-d u \partial\right),
$$

as quoted in Appendix A.2 where the embedding of the $d$ dimensional constant curvature algebra in the $d+1$ dimensional flat space algebra is described in detail. Hence, because

$$
\delta \phi=e^{-\left(\mathbf{N}_{d+1}-n / 2\right) u} \operatorname{Grad} e^{\left(\mathbf{N}_{d+1}-n / 2+1\right) u} \xi,
$$

\footnotetext{
${ }^{15}$ The higher spin Stückelberg formalism has been studied recently in [23].
} 
and the commutator $\left[N_{d+1}, \mathbf{G r a d}\right]=$ Grad, we obtain

$$
\delta \phi=\left[\operatorname{Grad}+\left(\mathbf{N}_{d+1}-n / 2\right) d u\right] \xi,
$$

where the second term on the right hand side appears because $[$ Grad, $u]=$ $d u$. To compute $\operatorname{Grad} \xi$ explicitly we note the following identities

$$
\begin{aligned}
{[\mathbf{g}, f(\mathbf{t r})] } & =4 \mathbf{t r} f^{\prime \prime}(\mathbf{t r})-4 f^{\prime}(\mathbf{t r})(\mathbf{N}+d / 2), \\
{[\operatorname{grad}, f(\mathbf{t r})] } & =-2 f^{\prime}(\mathbf{t r}) \mathbf{d i v}, \\
{[\mathbf{N}, f(\mathbf{t r})] } & =-2 \operatorname{tr} f^{\prime}(\mathbf{t r}) .
\end{aligned}
$$

So finally, orchestrating these instruments, a straightforward computation based on (74) and (75) gives the rather compact gauge variations

$$
\begin{aligned}
\delta \varphi_{0} & =\operatorname{grad} \xi_{1}+\mathbf{g} \xi_{2} \\
\delta \varphi_{1} & =\operatorname{grad} \xi_{2}+\left(\partial_{u}-[\mathbf{N}+1]-\frac{n}{2}+2-\mathbf{g} \operatorname{tr}\right) \xi_{1} \\
\delta \varphi_{2} & =-\operatorname{grad} \operatorname{tr} \xi_{1}+2\left(\partial_{u}-[\mathbf{N}+2]-\frac{n}{2}+3-\frac{1}{2} \mathbf{g} \operatorname{tr}\right) \xi_{2} \\
\delta \varphi_{3} & =-\operatorname{grad} \operatorname{tr} \xi_{2}-3\left(\partial_{u}-[\mathbf{N}+3]-\frac{n}{2}+4-\frac{1}{3} \mathbf{g} \operatorname{tr}\right) \operatorname{tr} \xi_{1},
\end{aligned}
$$

of the unconstrained $d$ dimensional fields $\varphi_{0}, \varphi_{1}, \varphi_{2}$ and $\varphi_{3}$ equivalent to that of the original $d+1$ dimensional field $\Phi$ in (70).

\subsection{Analysis of Gauge Transformations}

The gauge transformations (82)-(85) play a central rôle in what follows. As discussed in $[24,11]$, masses of bulk fields in de Sitter are related to conformal weights of boundary fields living on an initial Cauchy surface. Since we want to relate the operator $\partial_{u}$ to the mass by a Scherk-Schwarz reduction, we posit the relationship

$$
\partial_{u}=\Delta_{s}-\frac{n}{2}
$$

to be verified by the results that follow, where $\Delta_{s}$ obeys the spin $s$ massconformal weight relation

$$
m^{2}=-\Delta_{s}\left(\Delta_{s}-n\right)+(s-2)(s-2+n),
$$


discovered in [11]. Of particular interest are partially massless depth $t$ tunings of the conformal weight

$$
\Delta_{s}=n+s-t-1=\partial_{u}+\frac{n}{2} .
$$

If we fix the spin of $\varphi_{0}$ to be $s$ then the coefficients of the tracefree components of $\xi_{1}, \xi_{2}$ and $\operatorname{tr} \xi_{1}$ in (83)-(85) exactly reproduce the depth $t=1,2,3$ tuning relations, respectively. However, the origin of the remaining tunings for $t=4, \ldots, s$ are for now, obscure. Note however, that at depth $t$ these are governed by a traceless parameter $\bar{\xi}_{t}$ with index $\mathbf{N} \xi_{t}=(s-t) \bar{\xi}_{t}$ and mass tuning $\left(\partial_{u}+\mathcal{T}+\frac{1}{2}\right) \bar{\xi}_{t}=0$. (Note that traceless fields obey $\mathcal{N}=-\mathcal{T}$.)

Observe that when we Scherk-Schwarz identify $\partial_{u}$ with a generic mass parameter $\mu$, the $\xi_{1}, \xi_{2}$ gauge invariances are just shifts so that $\varphi_{2}, \varphi_{3}$ and the tracefree part of $\varphi_{1}$ can be gauged away ${ }^{16}$. I.e., these are Stückelberg fields. The massive models can be formulated in terms of a minimal covariant field content $\varphi \equiv \varphi_{0}$ and $\chi \equiv \frac{1}{\operatorname{tr} g} \operatorname{tr} \varphi_{1}$. However, for special values of the mass, the above transformations develop singularities corresponding to partially massless theories:

Partially massless gauge theories are based on higher derivative gauge invariances. At depth $t$ these take the form

$$
\delta \varphi_{0}=\left(\operatorname{grad}^{t}+\cdots\right) \bar{\xi}_{t},
$$

where the parameter $\bar{\xi}_{t}$ is traceless spin $s-t$. An important geometric result would be an explicit formula for "...": The trick is to find an appropriate field redefinition after which the gauge transformations can be written as a matrix with zeros everywhere save for its diagonal and next to diagonal entries. This allows us to readily identify degeneracies of the gauge transformations at special values of the mass parameter $\mu$ corresponding to partially massless theories. At other generic values, as discussed above, the gauge transformations are just Stückelberg shift symmetries. The required redefinition is

$$
\widetilde{\varphi}_{2}=\varphi_{2}+\frac{\mathcal{N}+\mathcal{T}}{\mathcal{N}+\mathcal{T}+2} \operatorname{tr} \varphi_{0}, \quad \widetilde{\varphi}_{3}=\varphi_{3}+\frac{\mathcal{N}+\mathcal{T}}{\mathcal{N}+\mathcal{T}+2} \operatorname{tr} \varphi_{1}
$$

which leads to gauge transformations

$$
\delta \widetilde{\varphi}_{2}=-2 \widetilde{\operatorname{grad}} \frac{\mathcal{T}-1}{\mathcal{T}\left((\mathcal{N}+2)^{2}-\mathcal{T}^{2}\right)} \operatorname{tr} \xi_{1}+2\left(\partial_{u}+\mathcal{T}+\frac{1}{2}\right) \xi_{2},
$$

\footnotetext{
${ }^{16}$ Actually, as discussed below, it is more convenient to gauge away linear combinations of $\varphi_{2}, \varphi_{3}$ and $\operatorname{tr} \varphi_{0}, \operatorname{tr} \varphi_{1}$.
} 
$\delta \widetilde{\varphi}_{3}=-2 \widetilde{\operatorname{grad}} \frac{\mathcal{T}-1}{\mathcal{T}\left((\mathcal{N}+2)^{2}-\mathcal{T}^{2}\right)} \operatorname{tr} \xi_{2}-2 \frac{\mathcal{N}+\mathcal{T}+3}{\mathcal{N}+\mathcal{T}+2}\left(\partial_{u}+\mathcal{T}+\frac{1}{2}\right) \operatorname{tr} \xi_{1}$

To appreciate the significance of these formulæ, expand the gauge parameters in traceless pieces

$$
\begin{aligned}
& \xi_{1}=\xi_{1}^{0}+\mathbf{g} \xi_{1}^{2}+\mathbf{g}^{2} \xi_{1}^{4}+\cdots, \\
& \xi_{2}=\xi_{2}^{0}+\mathbf{g} \xi_{2}^{2}+\mathbf{g}^{2} \xi_{2}^{4}+\cdots,
\end{aligned}
$$

and introduce a column vector $\left(\xi_{j}\right)=\left(\xi_{1}^{0}, \xi_{2}^{0}, \xi_{1}^{2}, \xi_{2}^{2}, \xi_{1}^{4}, \ldots\right)$ by identifying $\xi_{2 k+1}$ with $\xi_{1}^{2 k}$ and $\xi_{2 k+2}$ with $\xi_{2}^{2 k}$. (In general, we denote the expansion of a symmetric tensor $X$ into tracefree pieces $X^{2 k}$ by $X=X^{0}+g X^{2}+g^{2} X^{4}+\cdots$.) As mentioned above, the fields $\varphi_{1}^{0}, \widetilde{\varphi}_{2}$ and $\widetilde{\varphi}_{3}$ are Stückelberg at generic values of the mass and can be algebraically gauged away. However, expressing their gauge transformations as a matrix acting on the column vector $\left(\xi_{t}\right)$, we find

$$
\delta\left(\begin{array}{c}
\varphi_{1}^{0} \\
\widetilde{\varphi}_{2}^{0} \\
\widetilde{\varphi}_{3}^{0} \\
\widetilde{\varphi}_{2}^{2} \\
\vdots
\end{array}\right)=\left(\begin{array}{ccccc}
u_{1} & v_{1} & & & \\
& u_{2} & v_{2} & & \\
& & u_{3} & v_{3} & \\
& & & u_{4} & \ddots \\
& & & & \ddots
\end{array}\right)\left(\begin{array}{c}
\xi_{1}^{0} \\
\xi_{2}^{0} \\
\xi_{1}^{2} \\
\xi_{2}^{2} \\
\vdots
\end{array}\right) \equiv M \cdot\left(\xi_{t}\right) .
$$

where the diagonal entries are the tunings

$$
u_{j} \propto \partial_{u}+\mathcal{T}+\frac{1}{2}
$$

(Notice that by tracelessness we have $\left(\partial_{u}+\mathcal{T}+\frac{1}{2}\right) \bar{\xi}_{t}=\left(\partial_{u}-\frac{n}{2}-s+t+1\right) \bar{\xi}_{t}$ which agrees with (88).) Moreover the next to leading diagonal entries are simply $v_{j} \propto \widehat{\operatorname{grad}}$. Hence, whenever $\operatorname{det} M \neq 0$, i.e. in the case that the diagonal tunings $u_{j}$ do not vanish, the theory is described by a massive action

$$
S\left(\varphi \equiv \varphi_{0}, \varphi_{1} \equiv g \chi, \widetilde{\varphi}_{2}=0, \widetilde{\varphi}_{3}=0\right),
$$

with no gauge invariances remaining. However, whenever some

$$
u_{t}=0
$$


the matrix $M$ is degenerate. In that case we can still choose

$$
\left(\xi_{j}^{>}\right)=\left(\begin{array}{c}
0 \\
\vdots \\
0 \\
\xi_{t+1} \\
\xi_{t+2} \\
\vdots
\end{array}\right)
$$

so as to gauge away the corresponding lower components of $\widetilde{\varphi}_{2}, \widetilde{\varphi}_{3}$. We can then arrange for the action (95), with the same field content as in the massive case, to be gauge invariant by solving for a zero mode

$$
\left(\xi_{t}^{\leq}\right)=\left(\begin{array}{c}
\prod_{i=1}^{t-1}\left(-u_{i}^{-1} v_{i}\right) \cdot \bar{\xi}_{t} \\
\vdots \\
-u_{t-1}^{-1} v_{t-1} \bar{\xi}_{t} \\
\bar{\xi}_{t} \\
0 \\
0 \\
\vdots
\end{array}\right),
$$

of $M$ with a single, independent, traceless gauge parameter $\bar{\xi}_{t}$. This works because the upper components of $\widetilde{\varphi}_{2}, \widetilde{\varphi}_{3}$ and $\varphi_{1}^{0}$ do not transform under this gauge transformation. Specializing to spin $s$, there are precisely $s$ such degeneracies which correspond to partial gauge invariances of the generically massive action (95). We analyze these transformations and theories in depth in Section 6. (In particular, the zero mode (97) provides the promised "..." of (89).) A pressing task is the computation of the $d$ dimensional action $S\left(\varphi_{0}, \varphi_{1}, \widetilde{\varphi}_{2}, \widetilde{\varphi}_{3}\right)$ and its truncation (95). The latter action describes both massive and partially massless theories. We first illustrate the above ideas for the case of spin 2.

\subsection{Example: Spin 2}

Let us denote $\varphi_{0} \equiv h, \varphi_{1} \equiv A$ and $\varphi_{2}=\widetilde{\varphi}_{2} \equiv \sigma$, being a symmetric tensor, vector and scalar, respectively. The gauge symmetries (82), (83) and (91) 
read

$$
\begin{aligned}
\delta h & =\operatorname{grad} \xi_{1}+\mathbf{g} \xi_{2} \\
\delta A & =\operatorname{grad} \xi_{2}+\left(\partial_{u}-\frac{n}{2}\right) \xi_{1} \\
\delta \sigma & =-\operatorname{grad} \operatorname{tr} \xi_{1}+2\left(\partial_{u}-\frac{n}{2}+1\right) \xi_{2} .
\end{aligned}
$$

The massive theory is obtained by a Scherk-Schwarz reduction

$$
\partial_{u}^{2}+m^{2}=\frac{n^{2}}{4}
$$

In this case both $A$ and $\sigma$ are Stückelberg fields that can be algebraically gauged away by choices of $\xi_{1}$ and $\xi_{2}$. However, if we examine more carefully the matrix of gauge transformations for the candidate Stückelberg fields, i.e., equation (93), we have

$$
\delta\left(\begin{array}{c}
A \\
\sigma
\end{array}\right)=\left(\begin{array}{cc}
\partial_{u}-\frac{n}{2} & \operatorname{grad} \\
& 2\left(\partial_{u}-\frac{n}{2}+1\right)
\end{array}\right)\left(\begin{array}{l}
\xi_{1} \\
\xi_{2}
\end{array}\right),
$$

which is degenerate whenever

$$
\partial_{u}=\frac{n}{2} \text { or } \partial_{u}=\frac{n}{2}-1
$$

Inserting these relations in (99) (and reinstating the cosmological constant) gives masses

$$
m^{2}=0 \text { or } m^{2}=\frac{n-1}{n} \Lambda .
$$

(In dimension 4, the latter is the well-known $m^{2}=2 \Lambda / 3$ tuning for partially massless spin 2 fields $[25,26]$.) Hence, the zero eigenvectors in these two cases

$$
\left(\begin{array}{c}
\bar{\xi}_{1} \\
0
\end{array}\right) \text { or }\left(\begin{array}{c}
\operatorname{grad} \bar{\xi}_{2} \\
\bar{\xi}_{2}
\end{array}\right)
$$

correspond to gauge invariances

$$
\delta h=\operatorname{grad} \bar{\xi}_{1} \text { or } \delta h=\operatorname{grad}^{2} \bar{\xi}_{2}+\mathbf{g} \bar{\xi}_{2},
$$

of the theory after setting $A=\sigma=0$. The first invariance is the linearized diffeomorphism invariance of massless spin 2 fields (gravitons), while the second is the scalar gauge invariance of the partially massless spin 2 theory. 


\subsection{Radially Reduced Action}

Our major result is the massive, constant curvature, higher spin action obtained by radial reduction. The computation proceeds in the following steps:

(0) The starting point is the massless action in $d+1$ flat dimensions ${ }^{17}$ (obtained by letting $\Lambda \rightarrow 0$ in (58))

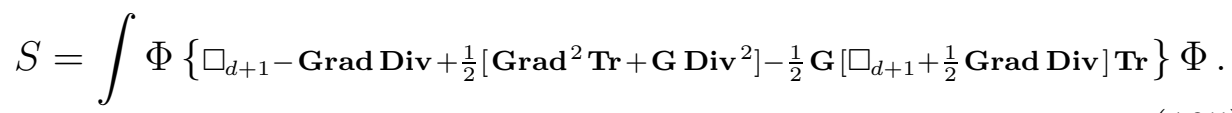

(i) We Weyl rescale the $d+1$ dimensional, real, massless field by

$$
\Phi \equiv e^{\left(\mathbf{N}_{d+1}-n / 2\right) u} \phi,
$$

as discussed above. All exponentials of $u$ and therefore explicit $u$ dependence cancels when we remember the $u$ dependence of the metric determinant, the dualization defined in (55) and the exponentials appearing in the embedded algebra (146). However, to achieve this end, we must first pass the Weyl exponentials through the operators Div and Grad according to

$$
\begin{aligned}
\operatorname{Grad} e^{\left(\mathbf{N}_{d+1}+\alpha\right) u} & =e^{\left(\mathbf{N}_{d+1}+\alpha-1\right) u}\left[\operatorname{Grad}+\left(\mathbf{N}_{d+1}+\alpha-1\right) d u\right], \\
\operatorname{Div} e^{\left(\mathbf{N}_{d+1}+\alpha\right) u} & =e^{\left(\mathbf{N}_{d+1}+\alpha+1\right) u}\left[\operatorname{Div}+\left(\mathbf{N}_{d+1}+\alpha+1\right) \partial\right]
\end{aligned}
$$

We may also now express operators of the $d+1$ dimensional flat space algebra in terms of $d$ dimensional constant curvature ones. (The factors $\exp \{ \pm 2 u\}$ appearing in (146) are handled as above.)

(ii) The action now takes the form

$$
S=\left[\int_{-\infty}^{\infty} d u\right] \int \phi \widetilde{G}\left(\mathbf{g}, \operatorname{grad}, \mathbf{N}, \square, \operatorname{div}, \mathbf{t r} ; d u, \partial, \partial_{u}\right) \phi,
$$

with no explicit $u$-dependence and the integration measure is the $d$ dimensional constant curvature one. Hence the operator $\partial_{u}$ is by now central. The one dimensional Heisenberg algebra $(d u, \partial)$ commutes with the $d$ dimensional constant curvature algebra. It is important to remember that the field $\phi$ and its dual depend on $d u$ and $\partial$ as indicated in $(75)$.

\footnotetext{
${ }^{17}$ The flat massless actions date back to the work of $[27,28,29]$.
} 
(iii) Next we must perform the $(\partial, d u)$ algebra in terms of the generalized Hadamard product defined in Section 2.2.1. Noting that $\partial f(d u \sqrt{\mathbf{t r}})=$ $\sqrt{\operatorname{tr}} f^{\prime}(d u \sqrt{\operatorname{tr}})$, this problem reduces to the following Hadamard products

$$
\begin{gathered}
(\cos * \cos )(x)=\cosh (x), \quad(\sin * \sin )(x)=\sinh (x), \\
(\cos * \sin )(x)=0=(\sin * \cos )(x) .
\end{gathered}
$$

(iv) At this point only $d$ dimensional constant curvature operators as well as $\partial_{u}$ (which can be expressed in terms of the mass by Scherk-Schwarz reduction of the log-radial momentum) are left, so the theory is now a $d$ dimensional one. However it still remains to make the field redefinition (90) explained in Section 5.2, a computation easily performed by first expressing (div, grad) in the Casimir basis ( $\widetilde{\mathbf{d i v}}, \widetilde{\text { grad }})$ via (50). The result, $S\left(\varphi_{0}, \varphi_{1}, \widetilde{\varphi}_{2}, \widetilde{\varphi}_{3}\right)$ is invariant under the Stückelberg gauge transformations (82), (83) and (91). It may be viewed as a gauge invariant, Stückelberg, formulation of the massive higher spin theory.

(v) The final step is to truncate to $S\left(\varphi_{0}, \chi\right)$ as described in the previous Section (by either gauging away Stückelberg fields or decoupling fields that do not transform under partial gauge variations) and at this final juncture Scherk-Schwarz reduce the log-radial momentum according to $^{18} \partial_{u}^{2} \equiv \mu^{2}$. At a fixed spin $s$ for which $(\mathbf{N}-s) \varphi=0=(\mathbf{N}-s+3) \chi$, the parameter $\mu$ is

$$
\mu^{2}=-m^{2}+\left(s+\frac{n}{2}-2\right)^{2}
$$

in terms of the more customary physical mass parameter $m$ (defined by requiring $m=0$ for strict masslessness).

\footnotetext{
${ }^{18}$ Hermiticity of the action, in which terms linear in $\mu$ appear, follows when the adjoint operation $\dagger$ acts on the parameter $\mu$ as it does for the derivative: $\partial_{u}^{\dagger}=-\partial_{u}$. If one prefers an action in which no imaginary parameters appear, the auxiliary field $\chi$ can be rescaled by a factor $i$, at the cost of a ghostlike sign for its kinetic term. This is legal because the auxiliaries are not part of the physical spectrum. Of course, the main difficulty of constructing higher spin interactions is ensuring that ghostlike auxiliaries are never physical excitations.
} 
The above computations are straightforward yet tedious. Their result is

$$
\begin{aligned}
& S=\varphi\left[\square+\mu^{2}-\left(\mathcal{N}+\frac{1}{2}\right)^{2}+2\left(\mathcal{N}-\frac{n}{2}+\frac{1}{2}\right)\left(\mathcal{N}+\frac{n}{2}-\frac{1}{2}\right)-\frac{1}{4}(\widetilde{\operatorname{grad}}-\mathbf{g} \widetilde{\operatorname{div}}) \frac{1}{\mathcal{N}^{2}} \widetilde{\operatorname{div}}\right] \delta_{\operatorname{tr}} \varphi \\
& +\varphi\left[\frac{4 \mathcal{T}\left(\mathcal{T}-\frac{1}{2}\right)}{(\mathcal{N}+\mathcal{T})(\mathcal{T}-1)}\left(\square+2\left[\mathcal{T}-\frac{n}{2}+\frac{1}{2}\right]\left[\mathcal{T}+\frac{n}{2}-\frac{1}{2}\right]-\frac{1}{4} \widetilde{\operatorname{grad}} \frac{\mathcal{T}-1}{\mathcal{T}\left(\mathcal{T}-\frac{3}{2}\right)(\mathcal{N}-\mathcal{T}+2)} \widetilde{\operatorname{div}}\right)\right. \\
& \left.-\left(\mu^{2}-\left[\mathcal{T}+\frac{1}{2}\right]^{2}\right)(\mathcal{N}-\mathcal{T}-1)\right] \frac{\left(\frac{\mathcal{N}-\mathcal{T}+1}{2}\right)_{\frac{\mathcal{N}+\mathcal{T}-2}{2}}}{\left(\frac{1}{2}\right)_{\frac{\mathcal{N}+\mathcal{T}-2}{2}}} \Pi_{\operatorname{tr}} \varphi \\
& +\left\{2 \varphi \mathbf{g}\left[-\widetilde{\operatorname{grad}} \frac{\mu-\mathcal{T}+\frac{3}{2}}{(\mathcal{N}+\mathcal{T}+2)}+\mathbf{g} \frac{\left(\mu+\mathcal{T}-\frac{3}{2}\right)(\mathcal{N}-\mathcal{T}+5)}{(\mathcal{N}-\mathcal{T}+2)(\mathcal{N}+\mathcal{T}+1)(\mathcal{N}+\mathcal{T}+4)} \widetilde{\operatorname{div}}\right]\right. \\
& +\chi\left[\frac{4 \mathcal{T}\left(\mathcal{T}-\frac{1}{2}\right)}{\mathcal{T}-1}\left(\square+2\left[\mathcal{T}-\frac{n}{2}+\frac{1}{2}\right]\left[\mathcal{T}+\frac{n}{2}-\frac{1}{2}\right]-\frac{1}{4} \widetilde{\operatorname{grad}} \frac{\mathcal{T}-1}{\mathcal{T}\left(\mathcal{T}-\frac{3}{2}\right)(\mathcal{N}-\mathcal{T}+2)} \widetilde{\operatorname{div}}\right)\right. \\
& \left.\left.-\left(\mu^{2}-\left[\mathcal{T}+\frac{1}{2}\right]^{2}\right)(\mathcal{N}-\mathcal{T}+2)(\mathcal{N}+\mathcal{T}+3)\right] \frac{\mathcal{N}-\mathcal{T}+2}{\mathcal{N}+\mathcal{T}+1}\right\} \frac{\left(\frac{\mathcal{N}-\mathcal{T}+5}{2}\right)_{\frac{\mathcal{N}+\mathcal{T}}{2}}}{\left(\frac{1}{2}\right)_{\frac{\mathcal{N}+\mathcal{T}}{2}}} \chi .
\end{aligned}
$$

This is the generating function for all massive actions. When $(\mathbf{N}-s) \varphi=$ $0=(\mathbf{N}-s+3) \chi$ it describes spin $s$ excitations. For generic masses, the physical spectrum is correct because there is a set of constraints whose leading terms are all $s$ possible powers of the divergence $\operatorname{div}^{t} \mathcal{G}+\cdots=0$ of the equation of motion $\mathcal{G}[7,8,9,10,11,12]$. The existence of these constraints is guaranteed because the action (110) arises from gauge fixing the Stückelberg variations (82), (83) and (91). At tuned values of the mass parameter, the constraints become the Bianchi identities of partially massless theories. Explicit formulæ for these follow directly from the partially massless gauge transformations presented in the next Section. Solving these constraints, leads finally to an on-shell description where $\chi=0$ and, at spin $s$,

$$
\left(\square-m^{2}+2(s-1)(s+n-2)\right) \varphi=0=\operatorname{div} \varphi=\operatorname{tr} \varphi,
$$

which is the usual description of a massive higher spin field. See [11] for a representation theoretic account.

\section{Partially Massless Theories}

The simplest formulation of partially massless fields is on-shell where one searches for residual gauge invariances of the massive field equations (111). 
These exist at tuned values of the mass as given in (88). This approach is described in Appendix B and is actually a new result.

For interactions, one is more interested in an off-shell formulation in terms of an action and accompanying gauge invariances, which we now describe. Essentially, all we need to do is reassemble equations (94)-(97) for degeneracies of the massive Stückelberg gauge transformations. Firstly, by ScherkSchwarz reduction, we focus our attention on the sector of fixed log-radial momentum $\partial_{u}=\mu$. Then, a depth $t$ degeneracy with traceless gauge parameter $\bar{\xi}_{t}$ appears at the tuning

$$
\left(\mu+\mathcal{T}+\frac{1}{2}\right) \bar{\xi}_{t}=0
$$

for gauge parameters

$$
\xi_{1}=\sum_{k=0}^{\left[\frac{t-1}{2}\right]} \mathbf{g}^{k}\left[\prod_{j=2 k+1}^{t-1}\left(\frac{\bar{u}_{j}^{-1} v_{j}}{j-t}\right)\right] \bar{\xi}_{t}, \quad \xi_{2}=\sum_{k=0}^{\left[\frac{t}{2}\right]-1} \mathbf{g}^{k}\left[\prod_{j=2 k+2}^{t-1}\left(\frac{\bar{u}_{j}^{-1} v_{j}}{j-t}\right)\right] \bar{\xi}_{t} .
$$

In these formulæ $u_{j}=(j-t) \bar{u}_{j}$ and

$$
\begin{aligned}
& \bar{u}_{j}=\left\{\begin{array}{cl}
1 & j=1 \\
2 & j=2,4,6, \ldots \\
2 j\left(\mathcal{T}-\frac{j}{2}+\frac{1}{2}\right) & j=3,5,7, \ldots,
\end{array}\right. \\
& v_{j}=\left\{\begin{array}{cl}
-\frac{1}{2} \widetilde{\operatorname{grad}} \frac{1}{\mathcal{T}} & j=1 \\
-2 \widetilde{\operatorname{grad}} \frac{\mathcal{T}-1}{\mathcal{T}} & j=2,3,4, \ldots,
\end{array}\right.
\end{aligned}
$$

where the products are ordered from left to right with increasing $j$. Then, with $\xi_{1}, \xi_{2}$ as stated, the partial gauge transformations read

$$
\begin{aligned}
\delta \varphi & =\operatorname{grad} \xi_{1}+\mathbf{g} \xi_{2} \\
& =-\frac{1}{2} \widetilde{\operatorname{grad}} \frac{1}{\mathcal{T}} \xi_{1}+\mathbf{g}\left(\xi_{2}-\frac{1}{2} \frac{1}{\mathcal{T}(\mathcal{N}-\mathcal{T}+2)} \widetilde{\operatorname{div}} \xi_{1}\right) \\
\delta \chi & =-\operatorname{tr} \xi_{1}+\frac{1}{\operatorname{tr} g} \operatorname{tr}\left(\operatorname{grad} \xi_{2}+\left(\mu-\mathcal{N}+\frac{1}{2}\right) \xi_{1}\right)
\end{aligned}
$$




$$
\begin{aligned}
& =-\left(1-\frac{\mu-\mathcal{N}-\frac{3}{2}}{(\mathcal{N}+2)^{2}-\mathcal{T}^{2}}\right) \operatorname{tr} \xi_{1} \\
& -\frac{1}{2}\left(\frac{1}{(\mathcal{N}+2)^{2}-\mathcal{T}^{2}} \operatorname{tr} \widetilde{\operatorname{grad}} \frac{1}{\mathcal{T}}+\frac{1}{\mathcal{T}(\mathcal{N}-\mathcal{T}+2)} \widetilde{\operatorname{div}}\right) \xi_{2} .
\end{aligned}
$$

\section{Examples}

\section{Depth $t=1-$ Strictly Massless Theory}

When $t=1$, we have $\xi_{2}=0$ and $\xi_{1}=\bar{\xi}_{1}$ is itself traceless. Hence $\chi$ does not vary and

$$
\delta \varphi=\operatorname{grad} \bar{\xi}_{1},
$$

is the usual massless gauge transformation. It is a gauge invariance of the action (110) when $\mu$ obeys the tuning condition (112). In terms of the usual physical mass parameter $m$ given in (109) this means $m=0$. For generic masses $m$ the action, being massive, is not gauge invariant but the field equations are subject to the corresponding single derivative constraint (see $[7,8]$ for a detailed explanation of how gauge Bianchi identities become constraints in massive models).

Observe that the only fields transforming under this gauge variation are the doubly tracefree components of $\varphi$, i.e., $\varphi$ obeying $\operatorname{tr}^{2} \varphi=0$. This means that we may set all other fields to zero. (In fact, since the decoupled fields are in danger of being ghostlike, unitarity forces us to do so.) Hence we obtain the usual description of a strictly massless higher spin gauge field in terms of a doubly traceless field and a gradient gauge transformation.

\section{Depth $t=2$}

This is the first example of a higher (quadratic) derivative gauge invariance. The tracefree part of $\xi_{2}=\bar{\xi}_{2}$ is the independent parameter and $\xi_{1}$ is determined by (113) to be

$$
\xi_{1}=-\frac{1}{2} \widetilde{\operatorname{grad}} \frac{1}{\mathcal{T}} \bar{\xi}_{2} .
$$

The fields transform as

$$
\delta \varphi=\operatorname{grad} \xi_{1}+\mathbf{g} \bar{\xi}_{2}
$$




$$
\begin{aligned}
& =\frac{1}{4} \widetilde{\operatorname{grad}}\left(\widetilde{\operatorname{grad}}+\frac{1}{2} \mathbf{g} \widetilde{\operatorname{div}} \frac{1}{\mathcal{N}-1}\right) \frac{1}{\mathcal{N}(\mathcal{N}+1)} \bar{\xi}_{2} \\
& +\frac{1}{2} \mathbf{g}\left(\frac{\square-\frac{1}{2}(n+1)(n-3)}{\mathcal{N}+1}+2 \mathcal{N}\right) \bar{\xi}_{2} \\
\delta \chi & =\frac{1}{4} \frac{1}{\mathcal{N}(\mathcal{N}+1)} \widetilde{\operatorname{div}} \bar{\xi}_{2} .
\end{aligned}
$$

For spin 2, the field $\chi$ is absent and we recover the famous partially massless transformation $\delta \varphi=\operatorname{grad}^{2} \bar{\xi}_{2}+\mathbf{g} \bar{\xi}_{2}$, or in pedestrian notation $[25,26]$

$$
\delta \varphi_{\mu \nu}=\left(D_{(\mu} D_{\nu)}+g_{\mu \nu}\right) \xi .
$$

Again, this is a gauge invariance of the action (110) when $\mu$ obeys (112) and otherwise implies a double derivative constraint. In terms of the usual, spin $s$, physical, mass parameter the tuning is at $m^{2}=2 s+n-5$.

Finally, in accordance with the degree of freedom counting arguments of [7], we may truncate most of the auxiliary fields because only the tracefree and doubly trace-free parts of $\chi$ and $\varphi$ transform.

\section{Fermions}

We define the space of symmetric spinors $\odot \mathcal{F}$ much as we did symmetric tensors. Elements of this space are spinor-tensor fields of the form:

$$
\odot^{s} \mathcal{F} \ni \Psi=\psi_{\mu_{1} \ldots \mu_{s}} d x^{\mu_{1}} \ldots d x^{\mu_{s}} .
$$

Again we make no restriction to elements of definite index content, so sums of elements with differing values of $s$ are permitted. All the operators that act on symmetric tensors, also act on symmetric spinors with minor differences. In particular,

$$
\partial_{\mu}: \mathcal{F} \rightarrow \mathcal{F}
$$

operates in the same way as it does on symmetric tensors. It doesn't act on the spinor tensor $\psi_{\mu_{1} \ldots \mu_{s}}$ and operates on the differentials as in (16). The covariant derivative acts as per equation (11). We use the Dirac matrices to construct several useful operators. 
- Gamma:

$$
\gamma \equiv \gamma_{\mu} d x^{\mu}
$$

Explicitly,

$$
\gamma \psi_{\mu_{1} \ldots \mu_{s}} d x^{\mu_{1}} \ldots d x^{\mu_{s}}=\gamma_{\mu_{1}} \psi_{\mu_{2} \ldots \mu_{s+1}} d x^{\mu_{1}} \ldots d x^{\mu_{s+1}} .
$$

- Gamma-trace:

$$
\gamma^{*} \equiv \gamma^{\mu} \partial_{\mu}
$$

This is the operator adjoint to $\gamma$. Explicitly,

$$
\gamma^{*} \psi_{\mu_{1} \ldots \mu_{s}} d x^{\mu_{1}} \ldots d x^{\mu_{s}}=s \gamma^{\mu} \psi_{\mu \mu_{2} \ldots \mu_{s}} d x^{\mu_{2}} \ldots d x^{\mu_{s}} .
$$

The operator $\mathbf{N}$ still counts the number or tensor indices, so its kernel, spinor fields, coincides with that of $\gamma^{*}$.

- Dirac operator:

$$
\not D \equiv \gamma^{\mu} D_{\mu} .
$$

This is the usual Dirac operator on spinors and spinor-tensor fields.

Due to the Dirac matrix term in the covariant derivative commutator (11) for spinors, it is convenient to modify the div and grad operators. We define

$$
\mathcal{D i v}=\operatorname{div}+\frac{i}{2} \gamma^{*}
$$

and

$$
\mathcal{G} \text { rad }=\operatorname{grad}+\frac{i}{2} \gamma,
$$

which results in the following commutation relation in terms of the old commutators for div and grad:

$$
[\mathcal{D} \mathbf{i v}, \mathcal{G} \mathbf{r a d}]=[\operatorname{div}, \operatorname{grad}]+i \not D .
$$

This definition is motivated by cosmological supergravity [30], where the underlying anti de Sitter superalgebra implies the appearance of a modified covariant derivative

$$
\mathcal{D}_{\mu}=D_{\mu}+\frac{1}{2} \sqrt{-\Lambda / n} \gamma_{\mu} .
$$

Since we work in de Sitter units $\Lambda=n$, explicit $i^{\prime} s$ appear in formulæ. Indeed, actions for de Sitter higher spin fermi fields lose hermiticity, even 
though the underlying physical excitations correspond to unitary, locally positive energy, representations of the de Sitter isometry group [11]. All our results can be easily translated to anti de Sitter space by reinstating the cosmological constant through dimensional analysis, taking care of imaginary units as explained above.

The commutation and anticommutation relations of the above operators with themselves and the bosonic operators mentioned earlier are summarized in Figure 2. All the old bosonic commutation relations carry over except the commutator of $\mathcal{D}$ iv and $\mathcal{G}$ rad.

Before we describe the commutator of $\mathcal{D} \mathbf{i v}$ and $\mathcal{G}$ rad in more detail, let us introduce two Casimirs and related operators that appear in this superalgebra.

1. Denote the old $\operatorname{sl}(2, \mathbb{R})$ Casimir $\mathbf{c}$ that commuted with $\mathbf{t r}, \mathbf{g}$, and $\mathbf{N}$ by $\mathbf{c}_{B}$ (" $B$ " for bosonic). The corresponding operator for fermions,

$$
\mathbf{c}_{F}=\mathbf{c}_{B}-\frac{1}{2}\left[\gamma^{*}, \gamma\right]+\frac{1}{2}(n+1),
$$

commutes with $\operatorname{tr}, \mathbf{g}, \mathbf{N}, \gamma$, and $\gamma^{*}$. (These operators form an so $(2,1 \mid 1)$ super Lie algebra.)

2. The quadratic Casimir is the fermionic version of the bosonic Lichnerowizc operator (denoted $\square_{B}$ and $\square_{F}$, respectively):

$$
\square_{F}=\square_{B}-\frac{1}{2}\left[\gamma^{*}, \gamma\right]+\frac{1}{2}(n+1) .
$$

As in the bosonic case, the flat space limit is the Laplacian $\Delta$. The operator $\square_{F}$ is central.

3. There is a further quartic Casimir $\mathcal{D}$ of this superalgebra first introduced in [7], which we shall call the "Lichnerowizc-Dirac operator", given by

$$
\mathcal{D}=(2 \mathbf{N}+n-1) \not D+2 i \mathbf{c}_{F}-2\left(\mathcal{G} \mathbf{r a d} \gamma^{*}+\gamma \mathcal{D} \mathbf{i v}\right)+2 \gamma \not D \gamma^{*}
$$

The Lichnerowizc-Dirac operator is also central - it commutes with every other generator. 


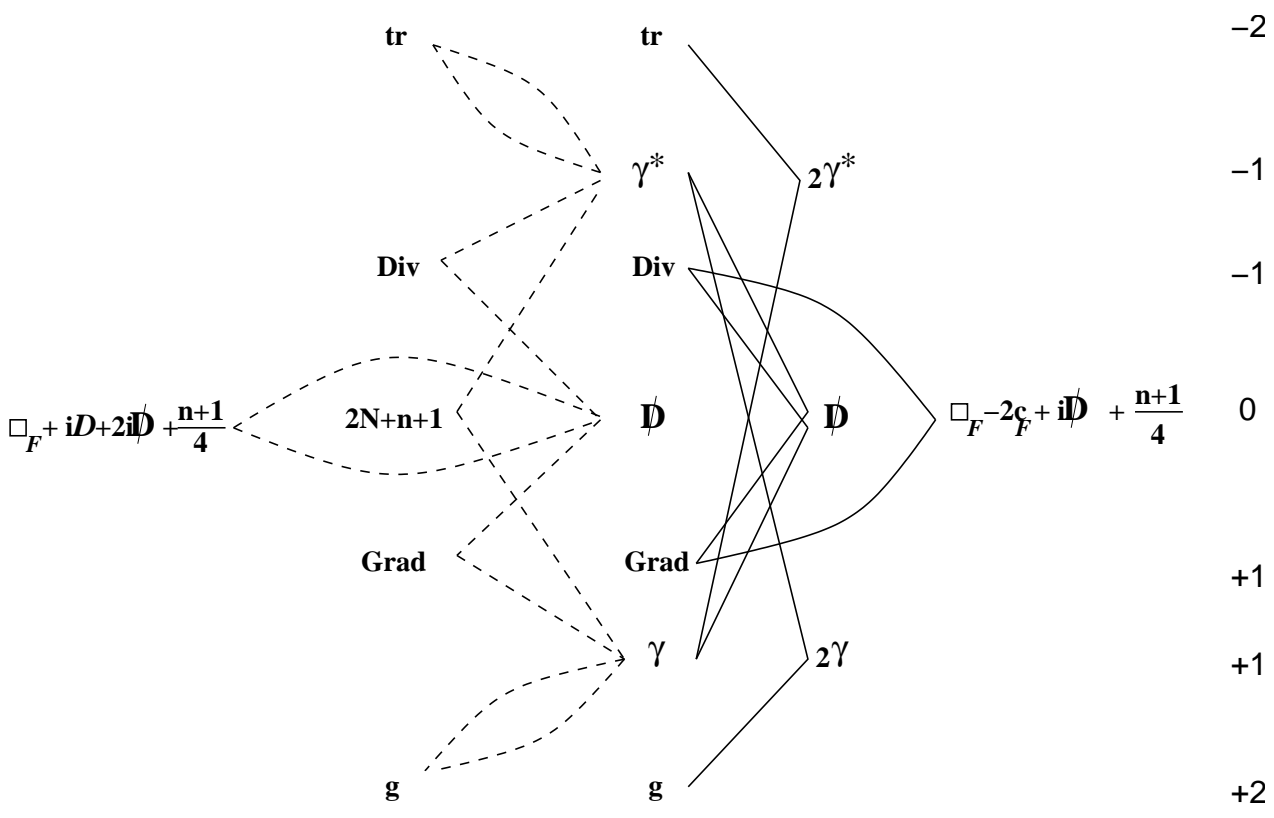

Figure 2: The algebra of operators on symmetric spinor tensors living on $n+1$ dimensional constant curvature manifolds. The right-hand column enumerates the weight with respect to the index operator $\mathbf{N}$. Only non-vanishing or results different from the bosonic case in Figure 1 are shown. Dashed lines denote anticommutators, while dashed loops are squares of operators (the diagram should be read from the middle column outwards). 
With the above technology on board, a simple expression for the $\mathcal{D}$ iv, $\mathcal{G}$ rad commutator, based on (11), follows

$$
[\mathcal{D i v}, \mathcal{G} \mathbf{r a d}]=\square_{F}-2 \mathbf{c}_{F}+i \not D+\frac{n+1}{4}
$$

In turn, the square of the Dirac operator reads

$$
\frac{1}{2}\{\not D, \not D\}=\square_{F}+i \mathcal{D}+2 i \not D+\frac{n+1}{4}
$$

The appearance of these various Casimirs is simply explained by employing a two component notation in which the $\operatorname{sl}(2, \mathbb{R})$ subalgebra action is made manifest. See Appendix A.3 for details. Also, a Casimir basis reformulation of the fermionic constant curvature algebra ought to exist, but we reserve its development for future study.

\subsection{Generating Function for Massless Fermion Actions}

We now give the half integer spin generalization of the massless, constant curvature action generating function. The minimum covariant field content for massless, half integer, higher spins is a traceless-gamma-traceless symmetric spinor-tensor $\psi$ :

$$
\gamma^{*} \operatorname{tr} \psi=0
$$

The correct degrees of freedom are imposed through the gauge invariance

$$
\delta \psi=\mathcal{G} \operatorname{rad} \xi
$$

Here, the parameter $\xi$ is gamma-traceless

$$
\gamma^{*} \xi=0
$$

The inner product is defined much as for the bosonic case

$$
\left\langle\xi_{1}, \xi_{2}\right\rangle=\int \sqrt{-g} \bar{\xi}_{1} \xi_{2}
$$

where the adjoint operation defined earlier for tensor operators is extended in the natural way to the fermionic case. 
The action for massless fields (assuming single derivatives) is then uniquely determined by the gauge invariance (138) which yields

$$
\begin{aligned}
S= & \int \bar{\psi}\left\{\mathcal{D}+i\left(\mathbf{N}^{2}+n \mathbf{N}-4\right)-i \mathbf{c}_{F}+\mathbf{g} \mathcal{D} \mathbf{i v} \gamma^{*}+\gamma \mathcal{G} \mathbf{r a d} \mathbf{t r}\right. \\
& \left.-\frac{1}{2} \mathbf{g} \not D \mathbf{t r}-i \gamma(4 \mathbf{N}+2 n-3) \gamma^{*}-\not D(2 \mathbf{N}+n-3)\right\} \psi \equiv \int \bar{\psi} \mathcal{R} \psi
\end{aligned}
$$

It is easy to check the Bianchi identity $\mathcal{D} \mathbf{i v} \mathcal{R}=0 \bmod \gamma$ corresponding to the gauge invariance (138).

\section{Scalar Fields on the Total Space}

The ideas in this Section apply to massive or massless theories in both flat and constant curvature spaces. However, for simplicity of presentation, we concentrate on the case of massless higher spins in $d$ dimensional Minkowski space $M$. The generating function for their actions is

$$
S=\int \Phi\left\{\square-\operatorname{Grad} \operatorname{Div}+\frac{1}{2}\left[\operatorname{Grad}^{2} \operatorname{Tr}+\mathbf{G}_{\operatorname{Div}^{2}}\right]-\frac{1}{2} \mathbf{G}\left[\square+\frac{1}{2} \operatorname{Grad} \operatorname{Div}\right] \operatorname{Tr}\right\} \Phi .
$$

Here the field $\Phi$ is subject to the double traceless constraint $\operatorname{Tr}^{2} \Phi=0$. Consider now the total space $E$ of the cotangent bundle $T^{*} M$ with coordinates $\left(x^{\mu}, d x^{\mu}\right)$. In the above action the field $\Phi=\Phi(x, d x)$ and may therefore be viewed as a scalar field on $E$. Hence, the theory of massless higher spins is reduced to a scalar theory in twice as many dimensions.

If we call $y^{\mu} \equiv d x^{\mu}$ our action becomes

$$
\begin{array}{r}
S=\int_{E} \Phi\left(x, \frac{\partial}{\partial y}\right)\left\{\square_{x}-y^{\mu} \frac{\partial}{\partial x^{\mu}} \frac{\partial}{\partial x_{\nu}} \frac{\partial}{\partial y^{\nu}}+\frac{1}{2}\left(\left[y \cdot \frac{\partial}{\partial x}\right]^{2} \square_{y}+y \cdot y\left[\frac{\partial}{\partial x} \cdot \frac{\partial}{\partial y}\right]^{2}\right)\right. \\
\left.-\frac{1}{2} y \cdot y\left(\square_{x}+\frac{1}{2} y^{\mu} \frac{\partial}{\partial x^{\mu}} \frac{\partial}{\partial x_{\nu}} \frac{\partial}{\partial y^{\nu}}\right) \square_{y}\right\} \Phi(x, y)
\end{array}
$$

where the integral over the total space $E$ is integration over the base space $M$ and setting $y$ to zero (after performing all possible $y$ derivatives).

Clearly this is a rather peculiar scalar field theory, since it has (i) a non-standard integration measure, (ii) a constraint $\square_{y}^{2} \Phi=0$ and (iii) a gauge invariance $\delta \phi=y^{\mu} \frac{\partial}{\partial x^{\mu}} \xi$. Moreover it is higher derivative and position dependent. These apparent drawbacks are a necessary price for handling all spins simultaneously. Possibly, a clever choice of gauge might lead to a tractable analysis, but we leave this issue to further study. 


\section{Conclusions}

This Article dealt with two main achievements. The first was a detailed analysis of the algebra of operators acting on the symmetric tensor bundle $\odot T^{*} M$ for an arbitrary manifold $M$. For general manifolds $M$, this algebra is a deformation of the semi-direct product $s l(2, \mathbb{R}) \times \mathbb{R}^{2}$ Lie algebra where the deformation appears in commutators of the $\mathbb{R}^{2}$ generators. In particular, by enlarging the universal enveloping algebra to include the square root of the $s l(2, \mathbb{R})$ Casimir and rational functions thereof, we displayed an operatorvalued diagonalization of the $s l(2, \mathbb{R})$ action on the $\mathbb{R}^{2}$ factor. This result holds already in the undeformed Lie algebra and provides an extremely useful calculus for universal enveloping algebra valued computations.

In the case that the underlying manifold $M$ is constant curvature, the deformation is extremely simple, given by the sum of the central Lichnerowicz wave operator and the quadratic $s l(2, \mathbb{R})$ Casimir. In some sense, we may view these results as the totally symmetric generalization of differential forms. In particular it should provide a powerful framework for future studies of constant curvature manifolds.

An obvious generalization of these results is to the tensor bundle $\mathcal{T} M$ of all possible tensors over the manifold $M$. These can be analyzed in terms of Young diagrams where each row corresponds to a symmetric tensor representation and in turn a copy of the algebra discussed above. This algebra must then be enlarged by operators mixing rows. We expect a similar picture to emerge, but leave this avenue to future studies.

A central physics application of totally symmetric tensor fields is the theory of higher spins-the second topic of this Article. Here the advances are as follows

- Computations involving symmetric tensors on curved manifolds are vastly simplified which is an important technical advance.

- Working in the space of all symmetric tensors we can write generating functions for all higher spin theories, rather than working at any given spin.

- We have shown how the Stückelberg formalism of [17] arises naturally via radial dimensional reduction. In particular, our formalism is in terms of a minimal set of unconstrained fields, rather than cumbersome towers of auxiliary fields. 
- Partially massless gauge transformations can be simply understood as degeneracies of the flat space transformation $\delta \Phi=\operatorname{Grad} \Xi$. This allows us to write explicit formulæ for partially massless gauge transformations at arbitrary spin.

We end with some more speculative remarks. Firstly, since our formalism deals naturally with arbitrary spins including infinite towers of higher spins, it seems that it should be well suited to studying the higher spin interaction problem. Indeed there are already indications $[14,13]$ that infinite towers of spins are a necessity for consistency of interactions. Moreover higher massive String states provide consistent couplings of infinite towers of higher spins. Clearly it would be very desirable to formulate interacting String dynamics within the approach we have presented, perhaps generalized to allow for tensors of mixed symmetry type, as discussed above.

Our final comment concerns the total space of the symmetric tensor bundle $\odot T^{*} M$. As remarked earlier we may view sections of this bundle as functions of coordinate differentials. Hence, as discussed in Section 8, higher spins can be described in terms of a novel, total space, scalar field theory. Clearly this approach deserves further investigation and also cries out for a String Field theoretic formulation.

\section{Acknowledgments}

It is a pleasure to thank Stanley Deser, Rod Gover, Andrew Hodge, Misha Khovanov, Sven Moch, Misha Movshev and Albert Schwarz for pertinent discussions. This work was supported by the National Science Foundation under grant PHY01-40365.

\section{A The Constant Curvature Algebra}

\section{A.1 Non-Commutative Harmonic Oscillators}

There is an interesting relationship between the constant curvature algebra and the harmonic oscillator. Begin with the flat space algebra and make the following identifications

$$
\operatorname{tr} \longleftrightarrow-\frac{1}{\hbar} p^{2}
$$




$$
\begin{aligned}
& \operatorname{div} \longleftrightarrow-i p \\
& \mathbf{N} \longleftrightarrow \frac{i}{2 \hbar}(x p+p x) \quad \square \longleftrightarrow \hbar \\
& \mathrm{g} \longleftrightarrow \frac{1}{\hbar} x^{2}
\end{aligned}
$$

Then, with the usual quantum commutator $[p, x]=-i$, the two algebras coincide. The right hand side is the spectrum generating $\operatorname{sl}(2, \mathbb{R})$ algebra of the harmonic oscillator with hamiltonian $2 H=\hbar(\mathbf{g}-\mathbf{t r})$ corresponding to the generator of the $S O(2)$ maximal compact subgroup.

This observation was not employed in our higher spin investigations because the harmonic oscillator representation diagonalizes the Casimir $\mathbf{c}=$ $\frac{1}{2} n(n-2)$, in particular there is no obvious constant curvature deformation. Nonetheless, it is conceivable that, using the reverse logic, the constant curvature algebra may provide an interesting non-commutative deformation of the harmonic oscillator algebra.

\section{A.2 Higher Dimensional Embedding}

The constant curvature algebra in dimension $d=n+1$, can be embedded in the flat space algebra of one dimension higher. This is achieved as follows: Introduce new operators $d u, \partial, \partial_{u}$ and $e^{u}$ with non-vanishing commutators

$$
[\partial, d u]=1, \quad\left[\partial_{u}, e^{u}\right]=e^{u} .
$$

Then, the flat space algebra $\left\{\operatorname{Tr}, \operatorname{Div}, \square_{d+1}, \mathbf{N}_{d+1}, \mathbf{G r a d}, \mathbf{G}\right\}$ in $d+1$ dimensions can be written in terms of its $d$ dimensional constant curvature counterpart as

$$
\begin{gathered}
\operatorname{Tr}=e^{-2 u}\left(\partial^{2}+\mathbf{t r}\right) \\
\mathbf{D i v}=e^{-2 u}\left(\mathbf{d i v}-\mathbf{t r} d u+\partial\left[\partial_{u}+n+1-d u \partial\right]\right) \\
\mathbf{N}_{d+1}=d u \partial+\mathbf{N} \quad \square_{d+1}=[\mathbf{D i v}, \mathbf{G r a d}] \quad \text { (central) } \\
\mathbf{G r a d}=\mathbf{g r a d}+\mathbf{g} \partial+d u\left[\partial_{u}-2 \mathbf{N}-d u \partial\right] \\
\mathbf{G}=e^{2 u}\left(d u^{2}+\mathbf{g}\right)
\end{gathered}
$$

Here

$$
\square_{d+1}=e^{-2 u}\left(\square+\partial_{u}^{2}+[n-2 N-2 d u \partial] \partial_{u}+2[\operatorname{grad} \partial-\operatorname{div} d u]\right.
$$




$$
\left.+\mathbf{g} \partial^{2}+\operatorname{tr} d u^{2}+d u(d u \partial-2 n) \partial-\mathbf{c}+\mathbf{N}(\mathbf{N}-n-1)\right),
$$

so it might seem remarkable that these complicated operators obey the simple flat space algebra - a point that is clear based, either on explicit computations or our discussion of radial dimensional reduction in Section 4.

We also note that there is the obvious relation between flat space algebras in adjacent dimensions

$$
\begin{array}{cc}
\mathbf{T r}=\partial^{2}+\mathbf{t r} & \mathbf{D i v}=\operatorname{div}+\partial \partial_{u} \\
\mathbf{N}_{d+1}=d u \partial+\mathbf{N} & \square_{d+1}=\square+\partial_{u}^{2} \\
\mathbf{G}=d u^{2}+\mathbf{g} . & \mathbf{G r a d}=\mathbf{g r a d}+d u \partial_{u}
\end{array}
$$

\section{A.3 Two Component Notation}

We can also define a two component notation for the constant curvature algebra that makes the $\operatorname{sl}(2, \mathbb{R})$ subalgebra manifest. This notation also generalizes to the fermionic superalgebra of Section 7 , so we concentrate on that case.

Let Greek indices $\alpha, \beta, \ldots=1,2$ label the fundamental representation of $\operatorname{sl}(2, \mathbb{R})$ and define

$$
f^{\alpha \beta}=\left(\begin{array}{cc}
\mathbf{g} & \mathbf{N}+\frac{n+1}{2} \\
\mathbf{N}+\frac{n+1}{2} & \mathbf{t r}
\end{array}\right)=f^{\beta \alpha}, \quad \epsilon^{\alpha \beta}=\left(\begin{array}{cc}
0 & 1 \\
-1 & 0
\end{array}\right)=\epsilon_{\alpha \beta},
$$

along with

$$
v^{\alpha}=\left(\begin{array}{c}
\mathcal{G} \text { rad } \\
\mathcal{D} \text { iv }
\end{array}\right), \quad \gamma^{\alpha}=\left(\begin{array}{c}
\gamma \\
\gamma^{*}
\end{array}\right) .
$$

Then $\operatorname{sl}(2, \mathbb{R})$ acts on a single index of an arbitrary two component vector as:

$$
\begin{aligned}
{\left[f^{\alpha \beta}, X^{\gamma}\right] } & =\epsilon^{\gamma \alpha} X^{\beta}+\epsilon^{\gamma \beta} X^{\alpha} \\
& =2 \epsilon^{\gamma(\alpha} X^{\beta)}
\end{aligned}
$$

which implies

$$
\left[f^{\alpha \beta}, f^{\gamma \delta}\right]=\epsilon^{\gamma \alpha} f^{\beta \delta}+\epsilon^{\gamma \beta} f^{\alpha \delta}+\epsilon^{\delta \alpha} f^{\gamma \beta}+\epsilon^{\delta \beta} f^{\gamma \alpha} .
$$


We also have

$$
\left\{\gamma^{\alpha}, \gamma^{\beta}\right\}=f^{\alpha \beta}
$$

as well as

$$
\begin{gathered}
{\left[\gamma^{\alpha}, v^{\beta}\right]=-\epsilon^{\alpha \beta} \not D \quad\left(\Rightarrow \not D=-\frac{1}{2}\left(\gamma^{\alpha} v^{\beta}-v^{\beta} \gamma^{\alpha}\right) \epsilon_{\alpha \beta}\right)} \\
\left\{\gamma^{\alpha}, \not D\right\}=2 v^{\alpha}
\end{gathered}
$$

Using $[\mathcal{D} \mathbf{i v}, \mathcal{G} \mathbf{r a d}]=v^{\alpha} \epsilon_{\alpha \beta} v^{\beta}$, we obtain explanatory formulæ for the various Casimirs

$$
\begin{aligned}
\mathbf{c}_{F} & =-\frac{1}{2} f^{\alpha \beta} \epsilon_{\beta \gamma} f^{\gamma \delta} \epsilon_{\delta \alpha}+\gamma^{\alpha} \epsilon_{\alpha \beta} \gamma^{\beta}+\frac{1}{4}(n+1)(n-3), \\
\square_{F} & =-v^{\alpha} \epsilon_{\alpha \beta} v^{\beta}-i \not D+2 \mathbf{c}_{F}-\frac{n+1}{4} \\
\mathcal{D} & =\gamma^{\alpha} \not D \epsilon_{\alpha \beta} \gamma^{\beta}+2 i \mathbf{c}_{F} .
\end{aligned}
$$

The higher spin actions we consider do not manifest this $\operatorname{sl}(2, \mathbb{R})$ symmetry, so little use was made of this elegant notation.

\section{B On-shell Partially Massless Fields}

There are various equivalent descriptions of partially massless fields. The simplest is the "on-shell" one. A spin $s$, depth $t$, on-shell, partially massless field is a section $\varphi$ of $\odot T^{*} M$ subject to

(i) $s$-index symmetric tensor:

$$
\mathbf{N} \varphi=s \varphi
$$

(ii) trace-free:

$$
\operatorname{tr} \varphi=0
$$

(iii) divergenceless:

$$
\operatorname{div} \varphi=0
$$


(iv) eigenmode of Laplacian ${ }^{19}$ :

$$
[\Delta+(\mathbf{N}-t)(\mathbf{N}+n-t-2)-\mathbf{N}-n+1] \varphi=0
$$

A generic eigenvalue of the Laplacian corresponds to a massive field. The above value is special because the system then enjoys a residual gauge invariance. For example, when $s=t=2$ we have

$$
[\square+n+1] h=0=\operatorname{div} h=\operatorname{tr} h .
$$

If we make a variation $\delta h=\left(\operatorname{grad}^{2}+\mathbf{g}\right) \bar{\xi}_{2}$ we find

$$
\begin{gathered}
{[\square+n+1] \delta h=\left(\operatorname{grad}^{2}+\mathbf{g}\right)[\square+n+1] \bar{\xi}_{2}, \quad \operatorname{div} \delta h=\operatorname{grad}[\square+n+1] \bar{\xi}_{2},} \\
\operatorname{tr} \delta h=2[\square+n+1] \bar{\xi}_{2} .
\end{gathered}
$$

Namely, the on-shell equations (157) enjoy a residual gauge invariance when $[\square+n+1] \bar{\xi}_{2}=0$. This equivalence relation of on-shell fields $h$ in turn implies the correct physical degree of freedom count $d(d+1) / 2-d-1-1=$ $d(d-1) / 2-2$.

The same computation can be performed in general. On-shell, the depth $t$, residual, partially massless gauge transformations are most simply given as

$$
\delta \varphi=\delta_{\text {tr }} \widetilde{\operatorname{grad}}^{t} \bar{\xi}_{t} .
$$

These transformations obviously obey the trace and Laplace conditions (ii) and (iv) whenever the parameter $\bar{\xi}_{t}$ does. Checking the divergence condition (iii) requires a more involved computation using the $\widetilde{\text { div }} \widehat{\text { grad }}$ relation (8). That it holds precisely is in fact an excellent cross check of that algebra.

\section{References}

[1] I. Bandos, X. Bekaert, J. A. de Azcarraga, D. Sorokin and M. Tsulaia, arXiv:hep-th/0501113.

[2] A. Lichnerowicz, Institut des Hautes Études Scientifiques, 10, 293 (1961); Bull. Soc. Math. France, 92, 11 (1964).

\footnotetext{
${ }^{19}$ Or for Lichnerowicz devotees $[\square+2 \mathbf{N}(\mathbf{N}+n-t-2)+(t+1)(t+1-n)] \varphi=0$.
} 
[3] C. M. Hull, JHEP 0109, 027 (2001) arXiv:hep-th/0107149.

[4] P. de Medeiros and C. Hull, Commun. Math. Phys. 235, 255 (2003) arXiv:hep-th/0208155.

[5] P. de Medeiros and C. Hull, JHEP 0305, 019 (2003) arXiv:hepth/0303036.

[6] L. P. S. Singh and C. R. Hagen, Phys. Rev. D 9, 898 (1974).

[7] S. Deser and A. Waldron, Nucl. Phys. B 607, 577 (2001) arXiv:hepth/0103198.

[8] S. Deser and A. Waldron, Phys. Rev. Lett. 87, 031601 (2001) arXiv:hepth/0102166.

[9] S. Deser and A. Waldron, Phys. Lett. B 508, 347 (2001) arXiv:hepth/0103255.

[10] S. Deser and A. Waldron, Phys. Lett. B 513, 137 (2001) arXiv:hepth/0105181.

[11] S. Deser and A. Waldron, Nucl. Phys. B 662, 379 (2003) arXiv:hepth/0301068.

[12] S. Deser and A. Waldron, arXiv:hep-th/0408155.

[13] L. Brink, R. R. Metsaev and M. A. Vasiliev, Nucl. Phys. B 586, 183 (2000) arXiv:hep-th/0005136.

[14] M. A. Vasiliev, space," arXiv:hep-th/0002183.

[15] T. Biswas and W. Siegel, JHEP 0207, 005 (2002) arXiv:hep-th/0203115.

[16] R. R. Metsaev, Phys. Lett. B 590, 95 (2004) arXiv:hep-th/0312297.

[17] Y. M. Zinoviev, arXiv:hep-th/0108192.

[18] M. Eastwood Bull. Belg. Math. Soc. Simon Stevin 11 (2005), 641.

[19] R. Manvelyan and W. Ruhl, arXiv:hep-th/0412252.

[20] J. Scherk and J. H. Schwarz, Nucl. Phys. B 153, 61 (1979). 
[21] C. Aragone, S. Deser and Z. Yang, Annals Phys. 179, 76 (1987).

[22] X. Bekaert, I. L. Buchbinder, A. Pashnev and M. Tsulaia, Class. Quant. Grav. 21, S1457 (2004) arXiv:hep-th/0312252.

[23] M. Bianchi, P. J. Heslop and F. Riccioni, arXiv:hep-th/0504156.

[24] L. Dolan, C. R. Nappi and E. Witten, JHEP 0110, 016 (2001) [arXiv:hep-th/0109096].

[25] S. Deser and R. I. Nepomechie, Phys. Lett. B 132, 321 (1983).

[26] S. Deser and R. I. Nepomechie, Annals Phys. 154, 396 (1984).

[27] C. Fronsdal, Phys. Rev. D 18, 3624 (1978).

[28] J. Fang and C. Fronsdal, Phys. Rev. D 18, 3630 (1978).

[29] T. Curtright, Phys. Lett. B 85, 219 (1979).

[30] P. K. Townsend, "Cosmological Constant In Supergravity," Phys. Rev. D 15, 2802 (1977). 